\section{5}

\title{
What it is like to be a bit: An Integrated Information Decomposition account of emergent mental phenomena
}

\author{
Andrea I. Luppi ${ }^{\mathrm{a}, \mathrm{b}}$, Pedro A.M. Mediano ${ }^{\mathrm{c}}$, Fernando E. Rosas ${ }^{\mathrm{d}, \mathrm{e}, \mathrm{f}}$, David J. Harrison ${ }^{\mathrm{g}, \mathrm{h}}$, Robin \\ L. Carhart-Harris ${ }^{\mathrm{d}}$, Daniel Bor ${ }^{\mathrm{c} *}$, Emmanuel A. Stamatakis ${ }^{\mathrm{a} *}$ \\ ${ }^{a}$ Division of Anaesthesia, School of Clinical Medicine, University of Cambridge, UK \\ ${ }^{b}$ Department of Clinical Neurosciences, University of Cambridge, Cambridge, UK

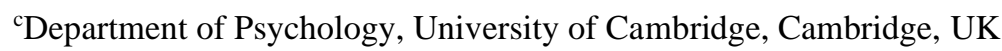
${ }^{\mathrm{d}}$ Center for Psychedelic Research, Department of Brain Science, Imperial College London, London, UK ${ }^{\mathrm{e} D a t a}$ Science Institute, Imperial College London, London, UK ${ }^{\mathrm{f}}$ Centre for Complexity Science, Imperial College London, London, UK ${ }^{\mathrm{g}}$ Department of History and Philosophy of Science, University of Cambridge, Cambridge, UK ${ }^{h}$ Leverhulme Centre for the Future of Intelligence, University of Cambridge, Cambridge, UK

*iThese authors contributed equally to this work. Correspondence: Andrea I. Luppi. Email: $\underline{\text { al857@ @am.ac.uk }}$

\section{Abstract:}

A central question in neuroscience concerns the relationship between consciousness and its physical substrate. Here, we argue that a richer characterisation of consciousness can be obtained by viewing it not as a monolithic construct, but rather as constituted of distinct information-theoretic elements. In other words, we propose a shift from quantification of consciousness - viewed as integrated information - to its decomposition. Through this approach, termed Integrated Information Decomposition (ФID), we lay out a formal argument that whether the consciousness of a given system is an emergent phenomenon depends on its information-theoretic composition - thus providing a principled answer to the long-standing dispute on the relationship between consciousness and emergence. Furthermore, we show that two organisms may attain the same amount of integrated information, yet differ in its information-theoretic composition. Building on $\Phi I D$ 's revised understanding of integrated information, termed $\Phi_{R}$, we also introduce the notion of $\Phi_{R}$-ing rate to quantify how efficiently an entity uses information for conscious processing. A combination of $\Phi_{\mathrm{R}}$ and $\Phi_{\mathrm{R}}$-ing rate may provide an important way to compare the neural basis of different aspects of consciousness. Thus, decomposition of consciousness enables us to identify qualitatively different 'modes of consciousness,' which establish a common space for mapping the phenomenology of different conscious states. We outline both theoretical and empirical avenues to carry out such mapping between phenomenology and information-theoretic modes, starting from a central feature of everyday consciousness: selfhood. Overall, Integrated Information Decomposition yields rich new ways to explore the relationship between information, consciousness, and its emergence from neural dynamics. 


\section{Introduction}

A central aim in both neuroscience and philosophy of mind is understanding the relationship between consciousness and its physical substrate. The past two decades have seen a proliferation of theoretical, experimental, and philosophical work on this topic (Crick \& Koch 2003; Friston 2012; Tononi \& Koch 2015; Koch et.al. 2015; Tononi et.al. 2016; Parr \& Friston 2019; Kleiner 2020). Additionally, an important and related question to how consciousness arises from matter concerns a mathematically precise and conceptually clear account of what constitutes 'emergence' (Tononi Sporns Edelman 1998; Friston et.al. 2014; Hesp et.al. 2019; De Caro \& Grasso 2017; Palacios et.al. 2020; Palacios et.al. 2019; Cea 2020). The present work is situated at the convergence of these two fundamental research avenues: that is, in this paper we seek to contribute to the formalisation of the relationship between consciousness and its underlying physical substrate, and elucidate to what extent this relationship can be rigorously understood in terms of emergence. To this end, we demonstrate the value of leveraging novel information-theoretic approaches to shed light on the nature of consciousness.

Here, we understand consciousness to be that, which is lost during dreamless sleep and deep anaesthesia, and recovered upon awakening (Schwitzgebel 2016). Following William James's celebrated notion of the stream of consciousness, we take the stance that subjective experiences dynamically unfold over time. For this reason, when seeking to understand the aspects of the physical world that correspond to subjective experiences, and give rise to them, we direct our attention to neural dynamics. Indeed, neural dynamics have recently been proposed as a "common currency" between brain and mind (Northoff et al., 2020), and a growing body of empirical work has demonstrated - through a variety of neuroimaging modalities and analytic approaches - that considering neural dynamics can provide powerful insights into consciousness and its alterations (Barttfeld et al., 2015; Deco et al., 2019; Demertzi et al., 2019; Dinesh et.al. 2020; Huang et al., 2020; Lee et al., 2019; Lord et al., 2019; Standage et al., 2020; Uhrig et al., 2018; Atasoy et.al. 2017; Atasoy et.al. 2018Luppi et.al. 2019; Luppi et.al. 2020bd; Vlisides et al., 2019; Varley et al., 2020a,b).

Thus, both theoretical and empirical reasons lead us to adopt a process-theoretic framework to the study of consciousness. Shannon's information theory is particularly well-suited to address process-theoretic approaches such as the one adopted here, because it enables the study of dynamical processes in a way that is not committed to any specific physical instantiation. This is not to say that the physicality of the system does not matter for the instantiation of consciousness: some material substrates may be more or less suitable (e.g., in terms of degrees of freedom) to sustain the dynamics on which consciousness depends (see esp. Barrett 2014). Rather, information theory enables us to focus on the dynamics and their properties, abstracted from considerations about physical instantiation or neuroimaging modality.

\section{From quantification of consciousness to its decomposition}

The close relationship between consciousness and information is a key aspect of the wellknown Integrated Information Theory of consciousness (hereafter, IIT) (Tononi et.al. 1994; Balduzzi \& Tononi 2008). A core intuition from IIT is that the "integration of information" represents a fundamental aspect of consciousness - and therefore, quantifying integrated information (suitably defined, as we explore in 'Technical preliminaries' below) enables an effective quantification of consciousness. 
Quantification is a key step in the scientific process, and the notion that consciousness could two decades. After quantification, decomposition into fundamental elements can be a fruitful next step in the scientific process, driving major advances in fields such as physics and chemistry over the last two centuries. As an illustrative example, knowing whether the clear liquid in one's glass is composed by twice as much hydrogen as oxygen, or oxygen and hydrogen in equal parts, represents the difference between drinking refreshing water or poisonous hydrogen peroxide. We believe that understanding the information-theoretic composition of consciousness will be similarly crucial for its scientific characterisation.

Within this context, the aim of this paper is to shed light on consciousness from an informationtheoretic perspective by capitalising on major advances in information theory, specifically within the recent framework of Partial Information Decomposition (PID) (Wibral et.al. 2017; Bertschinger et.al. 2014; Griffith \& Koch 2014; Lizier et.al. 2018) and its multivariate extension, Integrated Information Decomposition (IID) (Mediano et.al. 2019). Shannon's seminal results on information theory are mainly focused on information transmission between a single source and a single target; Partial Information Decomposition extends classic information-theoretic approaches to systems involving multiple sources of information (Wibral et.al. 2017). Moreover, the more recent development of Integrated Information Decomposition further extends PID to scenarios involving multiple targets as well as multiple sources (Mediano et.al. 2019). Such an extension is necessary if we are to investigate complex, multivariate systems with intricate causal relationships, of which the human brain is a prime example (Varley et al., 2020b; Turkheimer et.al. 2020). Thus, information decomposition - and $\Phi I D$ in particular - proves especially valuable for our purposes, being ideally suited to accommodate the multifaceted dynamics that characterise neural activity.

Here we propose a paradigm shift beyond the quantification of consciousness, and towards its decomposition into fundamental information-theoretic elements. For this purpose, we explore the consequences of viewing the core intuition that consciousness can be quantified in terms of integrated information in the light of Integrated Information Decomposition. (Note that we are not thereby committed to any of IIT's additional theoretical positions: for instance, we remain agnostic on whether integrated information is identical with consciousness, or coextensive with it under certain descriptions). Hence, a key goal of the present work is to demonstrate that understanding the composition of consciousness in terms of its informationdynamic building blocks may be crucial to obtain insights about what consciousness is - and the various modes in which it can be configured. Another major goal is the formal demonstration that consciousness can comprise both emergent and non-emergent phenomena - which can only be determined based on its information-theoretic composition.

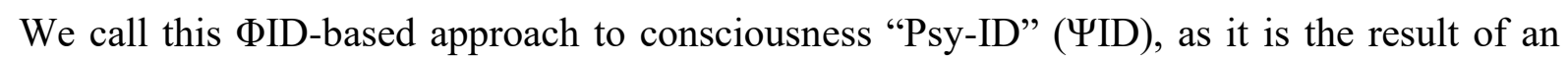
application of $\Phi I D$ to psychological phenomena. Importantly, this framework also sheds new light on other prominent theories of consciousness such as the Global Neuronal Workspace Theory (Dehaene \& Changeux 2011; Mashour et.al. 2020) by providing an informationtheoretic definition of the workspace in terms of synergistic interactions (Luppi et.al. 2020b). In contrast to other theoretical accounts of consciousness, YID provides a formal framework that is applicable to neuroimaging data, which enables a range of practical investigations on such observed dynamics (Mediano et.al. 2019; Luppi et.al. 2020a,b). This is especially important because we take it as imperative to our theoretical endeavours to provide predictions about consciousness that can be tested - and possibly falsified - by our current neuroimaging methods. 
The rest of this paper is structured as follows. First, 'Technical preliminaries' provides an accessible overview of the main information-theoretic intuitions and basic premises on which our work builds. Then, 'Theoretical contributions' introduces $\Psi$ ID to explicate the ramifications of ФID for understanding consciousness and the neural dynamics on which it depends. Therein, we specify core terminology that is relevant to the hypotheses and predictions we propose in the third section, 'Empirical predictions.' In this final section, we explore testable hypotheses and predictions as applied to recent experimental work on psychedelics and consciousness. This paper concludes with a series of remarks on the implications of $\Psi$ ID and its potential limitations.

\section{Technical preliminaries}

This section develops the formal intuitions and basic premises behind Integrated Information Theory, from which our paper takes its start. We then follow with an elaboration of the PID framework and its extension to IIT, called $\Phi I D$.

\section{The mind's $\Phi$ : Integrated Information Theory}

The starting point of our investigation is the notion that consciousness can be quantified in terms of the integrated information of a system - the fundamental intuition of Integrated Information Theory (Tononi 2004; Balduzzi and Tononi, 2008). Stemming from the seminal ideas put forward by Tononi, Sporns and Edelman (1994), IIT's core premise (which we share) is that the brain can be comprehensively characterized by its dynamics - i.e. the way in which the brain's activity unfolds over time (Tononi \& Edelman 1998; Northoff et al., 2020). Correspondingly, these dynamics allow the brain's current state to contain some information about its past and future states. Mathematically, this information that "flows" from the past to the future of a dynamical system can be captured by the so-called time-delayed mutual information (TDMI) (Barrett \& Seth 2011), denoted as $I\left(X_{t} ; X_{t+1}\right)$, where $X_{t}$ and $X_{t+1}$ indicate the state of the system at consecutive times $t$ and $t+1$. Broadly speaking, TDMI provides a first step towards quantifying the dynamical structure of a complex system (James et.al. 2011).

A further refinement on this idea is to consider not only the overall amount of information, but also its spatiotemporal distribution. Different parts of the brain exchange information constantly - and the existence and relevance of these precise informational patterns is uncontroversial in cognitive neuroscience. Moreover, although a precise, theoretically-based measure remains to be discovered, there is mounting evidence that the patterns of information that spread across the brain are related to consciousness (Casali et.al. 2013; Luppi et.al. 2020b).

One of the key insights of Tononi, Sporns and Edelman was to realise that this information must be shared across the brain in particular ways, such that it is simultaneously integrated (so the brain can behave as a whole) and differentiated (so its parts can perform independent processing). These ideas materialised into specific formulae to quantify integration and differentiation in the brain. In particular, the first such quantity to explicitly include the brain's dynamics into its formulation was the measure of integrated information proposed by Balduzzi \& Tononi (2008), 


$$
\Phi=I\left(X_{t} ; X_{t+1}\right)-\sum_{i=1}^{n} I\left(X_{t}^{i} ; X_{t+1}^{i}\right)
$$

167 As above, here $X_{t}$ denotes the state of the system as a whole at time $t ; X_{t}^{i}$ denotes the $i^{\text {th }}$ part of $168 X$ at time $t$ (and likewise for $X_{t+1}$ and $X_{t+1}^{i}$ ). Thus, this measure compares the information flow 169 between past and future (as captured by TDMI) observed in the whole system $X$, with the flow 170 observed within each of its parts - for this reason it is also referred to as "whole-minus-sum" $171 \Phi$ (Mediano et al., 2019). This measure is easy to compute (compared with other $\Phi$ measures) 172 (Oizumi et.al. 2016), and represents a noteworthy attempt to capture the powerful intuitions 173 behind IIT. However, this mathematical formulation has known shortcomings, including the 174 fact that it can yield negative values in some cases - which are hard to interpret, as a system cannot intuitively be "negatively integrated" or have negative consciousness (Oizumi et.al. 2016; Mediano et.al. 2018) . In the following, we show how these shortcomings can be overcome by means of the mathematical framework of Integrated Information Decomposition.

\section{Information decomposition}

179 The time-delayed mutual information on which $\Phi$ depends, is a special case of Shannon's mutual information, $I$. On a conceptual level, this "information" can be understood in several distinct but converging ways (Shannon 1948; Hellman \& Raviv 1970; Feder \& Merhav 1994; Jaynes 2003; Parrondo et.al. 2015). While extremely versatile, Shannon's information theory is (mainly) confined to interactions between pairs of variables (e.g. a sender and a receiver), and it is not equipped to study the information that multiple parts of a system have about each other's dynamical evolution.

186 Part of the necessary technical equipment for such analysis was introduced by Williams and 187 Beer (2010) with their framework of Partial Information Decomposition (PID). PID, in short, 188 decomposes the total information that two sources give about a target into four distinct "partial information atoms, " typically called synergistic, redundant, and unique information (note that for the sake of simplicity, throughout this work we will only consider the case of bivariate systems: for details of generalisation to more than two variables, we refer the reader to Mediano et. al (2019)).

As an illustrative example of redundant, unique and synergistic information, consider humans' two sources of visual information about the world: the eyes. The two eyes provide in part the same information, which is the information that is not lost when closing one or the other eye. This information is therefore carried redundantly by both sources. However, information about the very edge of the visual field is unique to each eye: this unique information is lost when the corresponding eye is closed. Finally, closing either eye will also remove stereoscopic information about depth: this information is not carried by either eye alone, but rather it requires both eyes together, arising synergistically from their interaction.

Thus, it is important to note that atoms are ways in which the information is being carried, and should not be confused with the sources of information that are doing the carrying, nor with the specific content that is being carried: any reference to e.g. "synergy" or "redundancy" should be understood as short-hand for "information carried synergistically" and "information carried redundantly," and likewise for other atoms. Therefore, as a way of example, if both my eyes show me that there is a red apple in front of me, then each eye (source) is carrying 
207 redundantly (atom) information about an apple (target), with the information in question being

208 that the apple is red (content).

209 By allowing us to distinguish qualitatively different phenomena involving multiple information

210 sources that cannot be disentangled by classic information theory (Rosas et.al. 2016), PID

211 moves beyond Shannon's information theory (James \& Crutchfield 2017). These novel

212 capabilities of PID have found fruitful application in multiple areas of neuroscience, from spike

213 trains to whole-brain dynamics (Stramaglia et.al. 2014; Wibral et.al. 2017).

\section{Decomposing information flow}

215 Thanks to the new lens provided by PID, it is now possible to decompose the information that

216 the past state of each part of the system, $X_{t}^{1}, \ldots, X_{t}^{n}$, carries about the future of the whole

217 system, $X_{t+1}$. Formally, for a system with $n=2$ parts, PID states that the information that part

$218 i$ has about the future of the system can be decomposed as

$I\left(X_{t}^{i} ; X_{t+1}\right)=\operatorname{Red}\left(X_{t} ; X_{t+1}\right)+\operatorname{Un}_{i}\left(X_{t} ; X_{t+1}\right)$,

220 where $\operatorname{Red}\left(X_{t} ; X_{t+1}\right)$ is the so-called redundant information provided by the sources (here, the

221 parts of the system), and $\operatorname{Un}_{i}\left(X_{t} ; X_{t+1}\right)$ is the information that $X_{t}^{i}$ provides uniquely. Similarly, the joint mutual information (i.e., information that the past state of the system as a whole carries about its future) is decomposed as

$I\left(X_{t} ; X_{t+1}\right)=\operatorname{Red}\left(X_{t} ; X_{t+1}\right)+\operatorname{Un}_{1}\left(X_{t} ; X_{t+1}\right)+\operatorname{Un}_{2}\left(X_{t} ; X_{t+1}\right)+\operatorname{Syn}\left(X_{t} ; X_{t+1}\right)$.

Here we see that in addition to redundancy and unique atoms, this expression includes by both sources jointly, but not separately (Fig. 1a). 


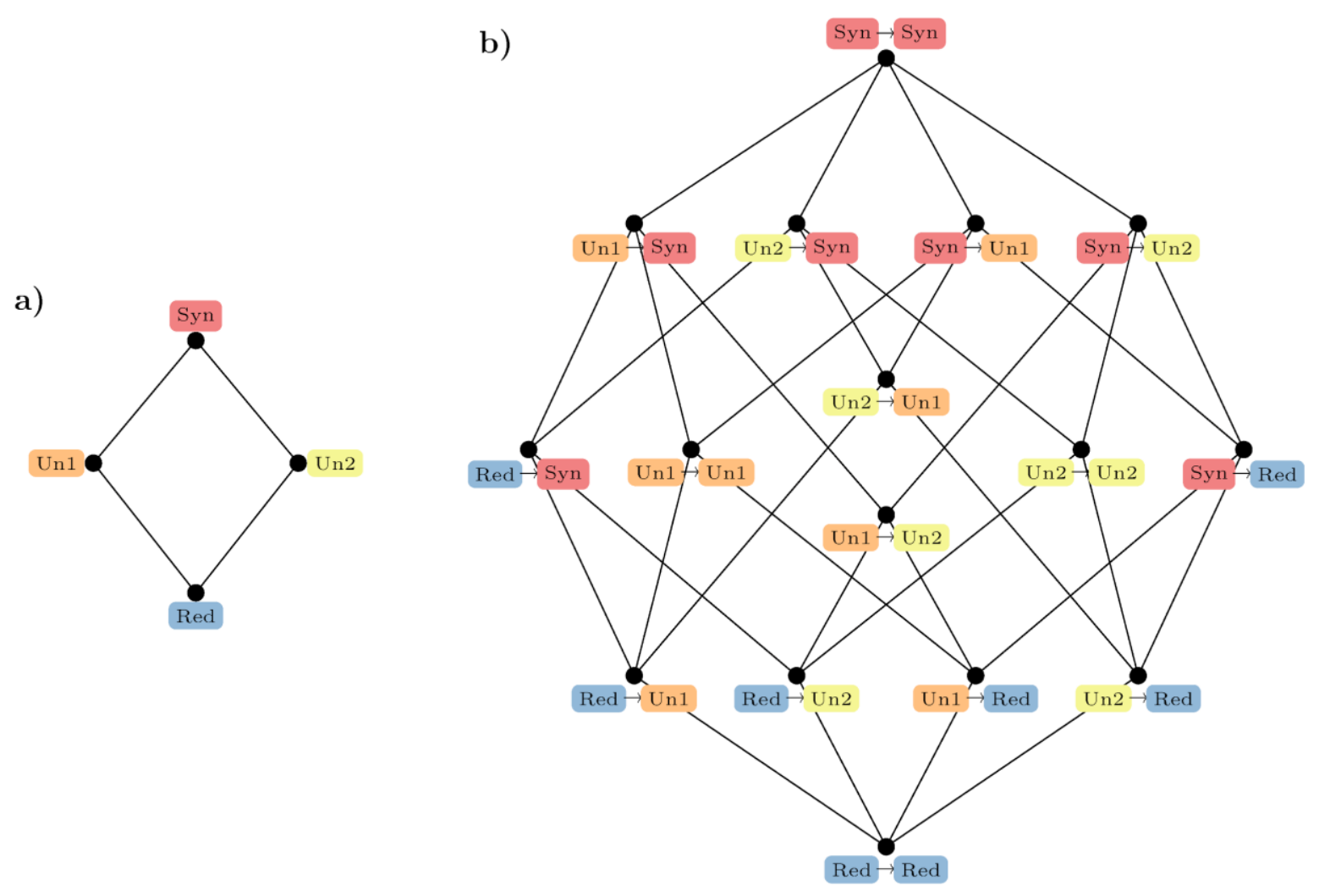

Figure 1. Visual representation of the relationships between PID and $\Phi I D$ atoms for bivariate systems. In both cases, to aid visualisation, atoms are arranged into a lattice, a mathematical construct which represents a hierarchical organisation of information content, such that redundant information (held by every variable) is at the bottom, and synergistic information (held only by the whole) is at the top. Please note that while here we focus in the case of two source variables, this information decomposition can be carried out over an arbitrary number of sources - which leads to a more elaborate lattice. For details of this more general construction we refer the reader to (Williams 2011).

Thus, PID provides the means to understand how each part of the system carries information about the future state of the system as a whole, decomposing this information flow into redundant, unique, and synergistic contributions. However, a key drawback of PID is that, while it is naturally applicable to multiple source variables, it can only be applied to scenarios with a single target. Thus, within the PID framework the future state of the system is still considered as a monolithic entity, without being able to consider the future state of each part individually. To resolve this shortcoming and extend the range of applicability of PID, Mediano et al. (2019) put forward the framework of Integrated Information Decomposition (ФID), which forms the mathematical basis of our proposal. Formally, ФID is a multi-target extension of PID, that can identify the redundant, unique, and synergistic components of the information that multiple source variables carry about multiple target variables.

Specifically, the information that is carried as each of the original four PID atoms (Red, Un , $U n_{2}$, and Syn, see Fig. 1a) at one point in time, at the next point in time may be carried as the same atom, or as any of the other atoms. Therefore, there are $4 \times 4=16$ combinations of PID atoms (Fig 1b), which correspond to the decomposition established by $\Phi I D$. For instance, $U n_{1} \rightarrow$ Syn denotes the information that is initially carried uniquely by the first source, and 
of the $\Phi I D$ atoms, and the generalisation to more than two variables, we refer the reader to

256 Mediano et. al (2019).

257 Taken together, PID and DID constitute valuable tools to refine our understanding of 258 information processing in dynamical systems - and, therefore, can be used to refine our theories of any phenomenon that depends on such information processing, like consciousness. In the same way as PID allows us to refine our understanding of mutual information, we will see that $\Phi I D$ allows us to refine our understanding of $\Phi$.

\section{Refining $\Phi$ through $\Phi I D$}

ФID makes it possible to decompose the information flow between the past and future states of each part of a system. Thus, a key feature of DID for our purposes is that it makes PID applicable to dynamical processes - including neural dynamics. A growing body of recent work has demonstrated that considering dynamical aspects of the brain can shed critical light on various aspects of consciousness (Northoff, 2020). For example, it has been shown that brain dynamics are significantly altered when consciousness is suppressed by anaesthesia or severe brain injury (Luppi et.al. 2019; Luppi et al., 2020; Huang et al., 2020; Demertzi et al., 2019; Barttfeld et al., 2015), or altered by psychedelics (Atasoy et al., 2017, Lord et al 2019, Luppi et al., 2020c, Luppi et al., 2020d). These results highlight the key role of neural dynamics for consciousness, vindicating the approach adopted by Tononi, Sporns, and Edelman (1998).

Specifically, $\Phi I D$ allows us to decompose the total information flow from past to future (TDMI, introduced above as $\left.I\left(X_{t} ; X_{t+1}\right)\right)$ into a range of modes of information dynamics, which can be used to deepen our understanding of various dynamical phenomena observed in the brain. A deeper understanding of $\Phi$ and its drawbacks, such as the reasons why it can adopt negative values, can be gained by decomposing it into its constituent information atoms.

279 Let's recall that TID decomposes the TDMI of a dynamical system with two parts into 16 280 disjoint atoms (illustrated in Fig. 1(b)). Of those 16 atoms, it can be shown mathematically 281 (Mediano et al., 2019) that 10 are represented in $\Phi$ (Fig. 2a): 7 correspond to all the synergistic information in the system, 2 correspond to the information transferred from one part to the other, and, importantly, one redundancy atom with a negative sign. Through this decomposition of $\Phi$ one can understand why $\Phi$ can sometimes take negative values: the subtraction of the redundancy atoms implies that $\Phi$ will be negative in redundancy-dominated systems, whenever redundancy is greater than the sum of synergy and information transfer. Note that transfer and synergy are rather different phenomena: while transfer refers to information that "moves" from one variable to another, synergy corresponds to phenomena that involve both variables, but cannot be seen in any of them when they are examined separately (Mediano et al., 2019).

290 Leveraging $\Phi I D$, a revised measure of $\Phi$, termed " $\Phi_{\mathrm{R}}$ ", can then be formulated by focusing on the synergy and transfer components and dropping the negative redundancy atom from the definition of integrated information (Mediano et al., 2019). Specifically, $\Phi_{\mathrm{R}}$ is the sum of synergistic and transfer atoms, and it is thereby guaranteed to be non-negative. Thus, the information decomposition of $\Phi$ provided by $\Phi$ ID can successfully identify the source of $\Phi$ 's theoretical difficulties, and provide a straightforward solution. In addition to the theoretical improvement (which will be further discussed below, in the section "A tale of ice and $\Phi_{\mathrm{R}}$ : From neural dynamics to the $\Phi_{\mathrm{R}}$-ing rate"), there is also recent evidence that $\Phi_{\mathrm{R}}$ also provides 


\section{1}

empirical advantages over the original formulation of $\Phi$ : unlike $\Phi, \Phi_{\mathrm{R}}$ is reduced between the same sets of brain regions both in patients with chronic disorders of consciousness, and during loss of consciousness induced by the intravenous anaesthetic, propofol (Luppi et al., 2020b). Importantly, reductions in $\Phi_{\mathrm{R}}$ were reversed when participants recovered consciousness after anaesthesia, demonstrating the relevance of $\Phi_{\mathrm{R}}$ for supporting human consciousness. Thus, given the theoretical advantages of $\Phi_{\mathrm{R}}$ over $\Phi$ outlined here, as well as these recently demonstrated empirical benefits (Luppi et al., 2020b), we will consider $\Phi_{R}$ as our primary metric of integrated information and conscious level throughout the rest of this work.

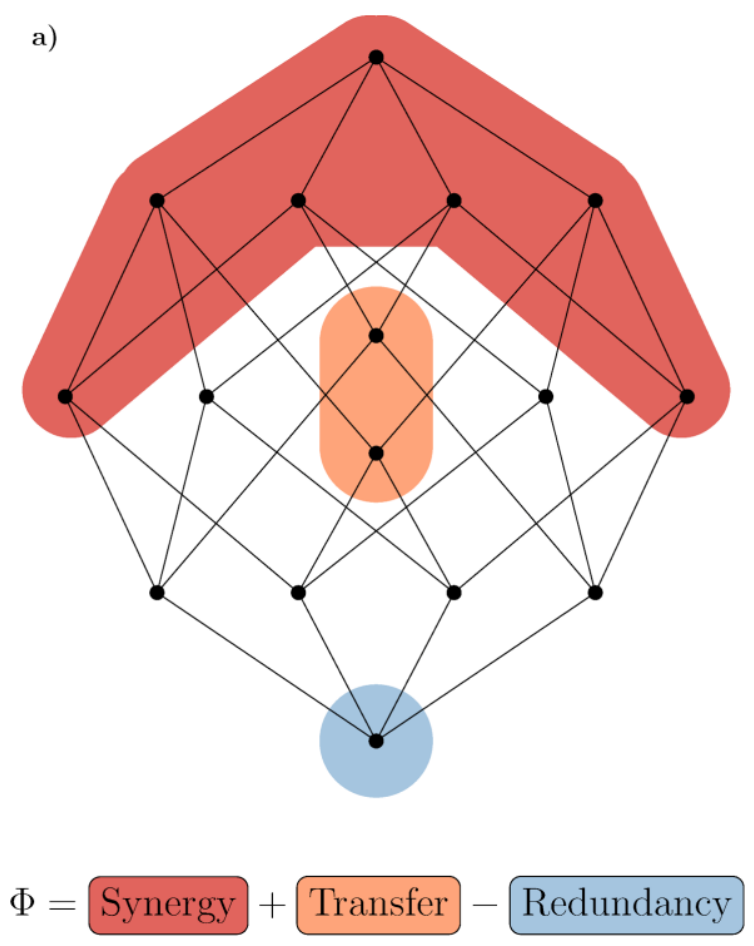

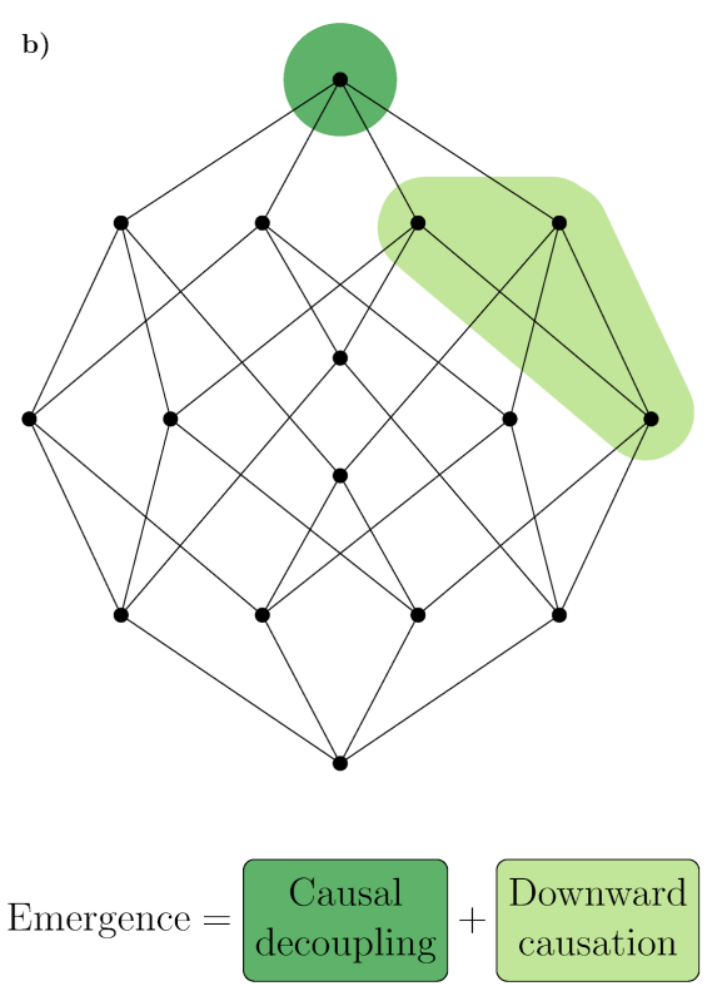

Figure 2. $\Phi I D$ lattice with relevant atoms for $\Phi(\mathbf{a})$ and causal emergence (b) highlighted in colour. 
Causal emergence is therefore defined here as the property of supervenient features to provide predictive power that cannot be explained from underlying microscale phenomena. In this manner, the coexistence of supervenience and irreducible predictive power of emergence - that have been previously thought as paradoxical (Bedau 1997, 2002) - can be resolved by operationalising supervenience in terms of instantaneous relationships between the system and its features, and identifying emergence with predictive power across time. Thus, a feature could be supervenient without being causally emergent, but not vice versa.

Crucially, the unique predictive power enabled by emergent features is quantified by $\Phi I D$. It is possible to prove that, under relatively general assumptions (Rosas et.al. 2020), a system's capacity to host causally emergent features depends directly on how synergistic are the system's dynamics. Furthermore, $\Phi I D$ allows us to distinguish two qualitatively different types of emergence: downward causation, in which an emergent feature has unique predictive power over individual parts; and causal decoupling, in which it has unique predictive power not over any constituent, but only over the system as a whole (Fig. 2b and Fig. 3).

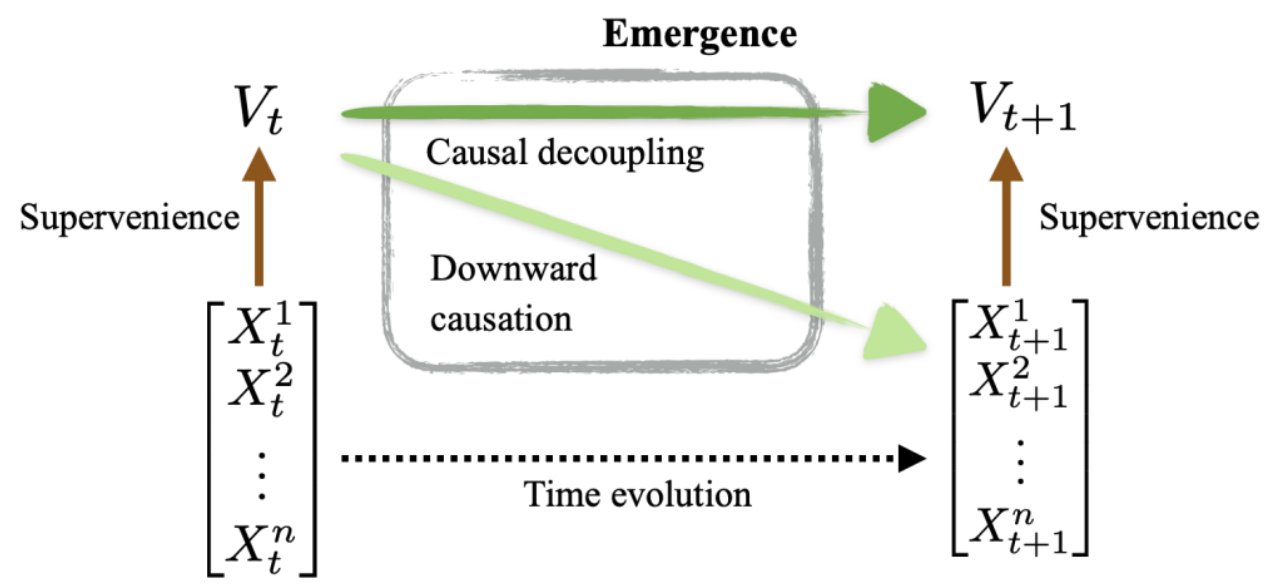

Figure 3. Causal decoupling and downward causation as two different types of emergent phenomena.

Interestingly, causal decoupling corresponds to the persistent synergies in the system (top atoms in the ФID lattice of Fig. 2b), which can be thought of as "the macroscale having causal influence on the macroscale, above and beyond the microscale effects" (Rosas et al., 2020) (Figure 2b). Microscale and macroscale are then related by downward (macro-to-micro) causation, as well as upward (micro-to-macro) causation, which respectively correspond to transformations of information from and to synergy. The distinction between supervenience and emergence (i.e., causal decoupling and downward causation) is also illustrated in Figure 3.

\section{Theoretical contributions: $\Psi$ ID}

To recapitulate, the aim of the present work is to provide a shift from the quantification of consciousness (viewed as integrated information) to its decomposition, and demonstrate how understanding consciousness in terms of SID atoms can shed light on several aspects of consciousness, including its relationship with emergence. If the close relationship between mental and neural dynamics assumed here turns out to be correct, and integrated information $\left(\Phi_{R}\right)$ happens to be a valid metric of conscious level, then our $\Psi$ ID framework is ideally suited 
to provide insight on the role of the different "modes" of neural dynamics - as determined by the $\Phi I D$ atoms - in supporting various aspects of mental phenomena.

\section{Rising $\Phi_{\mathrm{R}}$ : quantifying consciousness as an emergent property}

354

355

356

357

358

359

360

361

362

363

364

365

366

367

368

369

370

371

372

373

374

375

376

377

378

379

380

381

\section{2}

There has been abundant and vigorous philosophical debate over whether consciousness is an emergent phenomenon (Feinberg \& Mallat 2020). By (i) understanding consciousness as integrated information, (ii) decomposing it into its constituent information-theoretic atoms through $\Phi I D$, and then (iii) viewing those atoms in terms of causal emergence, we are in a position to establish a grounded, formal, and falsifiable way to address this problem representing the first core contribution of this paper.

In order to fully develop this argument, let's start noting that the $\Phi I D$ decomposition of $\Phi_{R}$ in Figure $2 \mathrm{a}$ and of emergence in Figure $2 \mathrm{~b}$ shows that consciousness (quantified by $\Phi_{\mathrm{R}}$ ) comprises the atoms of emergence among its constituents, but also comprises additional atoms that are beyond the scope of emergence. The implications of this mathematical fact are profound and far-reaching. In effect, note that nothing in IIT or $\Phi$ ID mandates that a given system should exhibit all atoms that constitute $\Phi_{R}$ in order to be conscious: all that is required is the presence of at least one of $\Phi_{\mathrm{R}}$ 's constituent atoms with non-zero value. Following this rationale, we can draw two conclusions about the relationship between consciousness and emergence:

1. Consciousness (integrated information) can be an emergent phenomenon: there could be systems whose $\Phi_{\mathrm{R}}$ derives (entirely or in part) from emergent $\Phi$ ID atoms.

2. Consciousness doesn't have to be emergent: there could also be systems whose $\Phi_{R}$ derives entirely from $\Phi I D$ atoms that do not enter into the composition of emergence (e.g. information transfer, or non-emergent synergy atoms).

Therefore, thanks to our information-decomposition approach to both consciousness and emergence, we can now see that the original question "Is consciousness an emergent phenomenon" was fundamentally ill-posed. Specifically, it was ill-posed because neither consciousness nor emergence are monolithic constructs, but rather they are both constituted by multiple information-theoretic atoms, and - crucially - only some of the atoms that constitute integrated information are emergent. Once this is understood, the answer becomes clear: some possible kinds of consciousness are emergent, others are not, and others may comprise both emergent and non-emergent atoms - in a way that is amenable to empirical investigation.

\section{A tale of ice and $\Phi_{\mathrm{R}}$ : From neural dynamics to the $\Phi_{\mathrm{R}}$-ing rate}

Decomposing $\Phi_{\mathrm{R}}$ can also provide another important insight about the nature of consciousness. Since not all atoms enter into the composition of $\Phi_{\mathrm{R}}$, dynamical processes with the same total amount of information flow (quantified by the time-delayed mutual information, TDMI) can still differ in their $\Phi_{R}$, and - conversely - organisms with the same $\Phi_{R}$ can differ in TDMI (Figure 4). The proportion of TDMI that is accounted for by $\Phi_{R}$, which we may refer to as the $\Phi_{R}$-ing rate, can then be thought as quantifying the efficiency with which the organism transforms information into consciousness - its "nougenic rate". In other words, cognitive architectures can differ in the "consciousness bang" that they provide for a given "informational buck." 


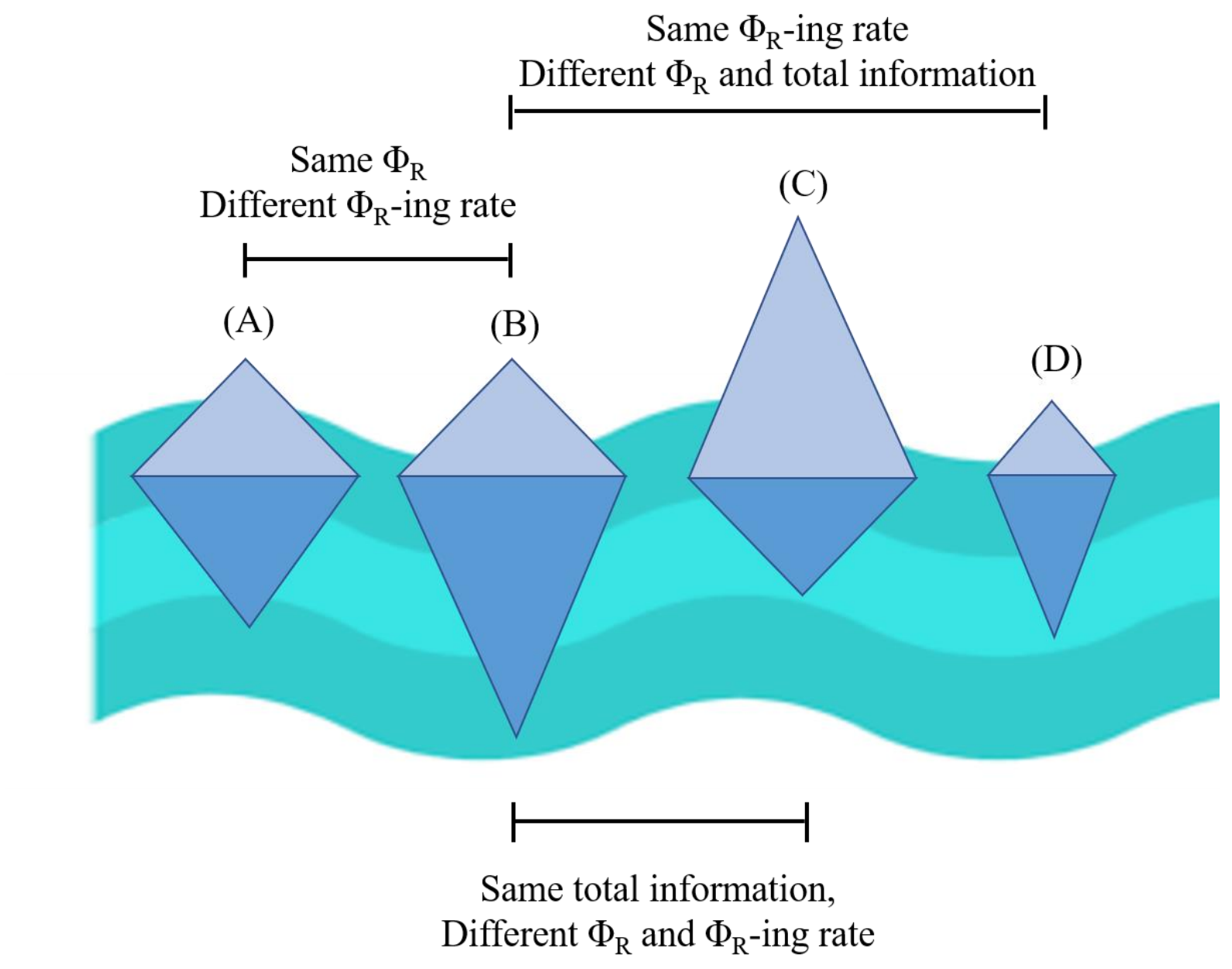

Figure 4. Differences in $\Phi_{\mathrm{R}}$ and $\Phi_{\mathrm{R}}$-ing rate. Four conscious beings are schematically depicted as 394 icebergs, with their total size representing their total information (TDMI), the part above water (light blue) representing the combination of atoms that compose $\Phi_{R}$, and the part below water being the information that does not contribute the $\Phi_{\mathrm{R}}$ (and hence to consciousness). Different beings can have the same $\Phi_{\mathrm{R}}$ despite different total information (A and B), or same total information but different $\Phi_{\mathrm{R}}$ and $\Phi_{R}$-ing rate (B and C), or different total information and different $\Phi_{R}$ but same $\Phi_{R}$-ing rate (B and D). Only considering $\Phi_{R}$ would ignore the difference between A and B, as well as the similarity between $\mathrm{B}$ and D.

Note that the $\Phi_{R}$-ing rate is in one respect closer than $\Phi_{R}$ to the original formulation of $\Phi$, in terms of scaling negatively with redundancy. Original $\Phi$ explicitly removed redundancy from the quantification of integrated information, but this led to the theoretical flaw that the resulting quantity was not guaranteed to be lower-bound by zero, and could instead be negative if redundancy dominated the system (see Section Refining $\Phi$ through $\Phi I D$ ). Being predicated as the ratio of $\Phi_{R}$ to TDMI, the $\Phi_{R}$-ing rate implicitly takes redundancy into account, as a component of TDMI; in fact, the more redundancy there is in a system, the lower the $\Phi_{\mathrm{R}}$-ing rate will be even if $\Phi_{R}$ is high overall. In other words, both $\Phi$ and the $\Phi_{R}$-ing rate acknowledge that there is an intuitive difference between a system where much of the information is redundant, and one that instead prioritises dynamics related to consciousness $\left(\Phi_{R}\right)$, and is 412 therefore efficient in its ability to turn information into consciousness. The relationship between $\Phi$ and $\Phi_{\mathrm{R}}$ is illustrated in Figure 4. 
414 At this stage we will not take a stance on whether the part of TDMI that does not contribute to

$415 \Phi_{\mathrm{R}}$ (e.g. the redundancy) is to be completely ignored from the study of consciousness - other

416 than as contributing to the calculation of the $\Phi_{\mathrm{R}}$-ing rate - or whether perhaps it could be

417 regarded as "preconscious" or "potential consciousness."

418 Thus, we argue that it can be beneficial to characterise information-processing systems with 419 both $\Phi_{R}$ and $\Phi_{R}$-ing rate, since they provide complementary views on integration information.

420 Therefore, it should be clear that our proposed $\Phi_{\mathrm{R}}$-ing rate is not intended to replace $\Phi_{\mathrm{R}}$, but 421 rather to complement it, thereby enabling a more encompassing framework for quantifications 422 of consciousness.

\section{Carved from $\Phi_{\mathrm{R}}$ : Carving consciousness at its joints}

424 Following a similar reasoning, an even more fundamental observation afforded by information decomposition is that even when two systems have the same $\Phi_{R}$ and the same $\Phi_{R}$-ing rate, they may be entirely different in the composition of their $\Phi_{R}$. In fact, no single $\Phi I D$ atom is essential for $\Phi_{\mathrm{R}}$, suggesting that two systems may have the same $\Phi_{R}$ despite not sharing any of its constituent atoms (e.g., a purely emergent system versus a transfer-only system). This possibility raises the fundamental question of whether different $\Phi I D$ atoms may correspond to different ways of being conscious.

Implicitly, IIT is committed to an affirmative answer to this question: some $\Phi I D$ atoms - it posits - are experienced and contribute to the system's consciousness (integrated information), while other atoms are not: there is nothing it's like to instantiate those atoms. This stance, however, acknowledges only the coarser aspects of being conscious: namely, being conscious or not at all. In contrast, IID suggests a richer perspective on this issue, by raising the possibility that different information-theoretic atoms may correspond to fundamentally different 'ways of being conscious' - or, as we call them, modes of consciousness: broad-strokes aspects of how consciousness is subjectively experienced, which may be more or less extended in time.

Illustrative examples of specific modes of consciousness may include the selflessness experienced during deep meditation or under the effects of psychedelic drugs; or the general way that depression may feel to those suffering from it (see Colombetti \& Ratcliffe 2012; Seth $\&$ Friston 2016 ; Deane 2020; Deane et al., 2020). Therefore, this is not the same as the notion of "qualia", which instead identify specific and fine-grained aspects of individual conscious experience (the redness of a particular apple; the painfulness of a stubbed toe). Although an IIT-based interpretation of qualia in terms of information geometry and category theory exists (Balduzzi \& Tononi 2009; Tsuchiya et.al. 2016; Moon \& Pae 2019) and a reinterpretation of this issue through the lens of IID may be possible, this is not our aim here. Note also that although empirical evidence suggests that different cognitive functions rely on different information-theoretic atoms (Luppi et.al. 2020a), our focus here is on altered states of consciousness, rather than normal fluctuations within day-to-day cognitive states.

In other words, YID contends that beyond the total amount of integrated information in an organism, additional insight may be gleaned from knowing its specific information-theoretic composition, which can be investigated through $\Phi I D$. By investigating the phenomenology associated with specific atoms, these "modes of consciousness" may provide a way to 
eventually understand the phenomenology of different states of consciousness from the thirdperson perspective - as explained below.

To demonstrate how $\Psi$ ID can enrich our understanding of consciousness, we contend that if integrated information is an effective measure of conscious level, then the corollaries obtained from YID would bring us one step closer to addressing the famous conundrum that has dogged the philosophy of mind: "What it is like to be a bat". That is, even though we elect to use the term modes of consciousness, and this term is different in its extension from the debate on qualia (for which Nagel's paper (1974) is typically placed as the cornerstone), it may still allow progress in that matter.

The alchemists of old sought to turn lead into gold, and failed mainly because their understanding of these substances reached only the level of their surface properties (shiny or dull, yellow or grey). Today, thanks to our understanding of atomic composition, turning lead into gold via nuclear transmutation is feasible (though not financially wise). Likewise, YID may represent a similar change of reference frame for our understanding of consciousness: rather than talking of "human consciousness" or "bat consciousness" tout court (like alchemists conceived of gold and lead as "substances"), we propose that it may be a more proficuous avenue to consider their respective atomic constitutions in terms of $\Phi I D$, and think in terms of information atoms rather than "molecules." Put differently, rather than trying to perform the mental "human-to-bat" conversion all at once, as Nagel proposed, an atomic understanding of integrated information may provide us with a Rosetta stone to guide a more nuanced comparison.

More generally, we claim that thinking in terms of 'modes' or 'kinds' of consciousness may be advantageous for the exploration of conscious states within and potentially also across organisms. From this point of view, the work of Nagel may be seen as shaping the debate too narrowly, focusing the discussion on fine-grained aspects of being a bat (flying through the air, echolocating its prey, etc); when it is unclear how much progress can be made from such a starting point towards truly understanding 'what it's like to be a bat'. We contend that failure to solve the question on Nagel's terms should not prescribe scientific pessimism, as it does not follow that every aspect of bat consciousness is foreclosed from investigation. In fact, we claim that while the fine-grained details related to contents associated with certain qualia may be highly specific - and hence possibly incommensurable, one might still be able to compare the broad-stroke modes. Indeed, if our approach proves viable, then there may be relevant inferences to be drawn in comparing quantified modes of consciousness via their corresponding $\Phi I D$ atoms between different systems, which could lead to insightful phylogenetic or cognitive similarities between conscious creatures.

497 To illustrate this point, let us focus on the challenge of seeking a $\Phi I D$ atomic decomposition 498 of "what it is like to be a human experiencing an altered state of consciousness," which can be 499 thought as a first step paving a road towards addressing conscious experiences of other species. 500 An atomic understanding of what it may be like to be in a given state of altered consciousness 501 is based on three elements. 
1. A description of the atomic composition of the integrated information generated by the human cognitive architecture under baseline conditions, to act as a reference point (although it should be noted that the question of defining a "baseline human state" is itself not trivial, as even so-called "resting state" may be better understood as "Random Episodic Spontaneous Thought") (Breakspear et al., 2004).

2. A description of the atomic composition of the integrated information generated in a particular state of interest. (If no $\Phi_{\mathrm{R}}$ is generated, then the question would be solved with the trivial answer "nothing", which might be the case for the alterations of consciousness induced by anaesthesia or disorders of consciousness, and indeed there is ongoing empirical research on this topic; see Luppi et.al. 2020b).

3. An understanding of what kind of changes in consciousness may correspond to each observed change of the atoms that constitute $\Phi_{\mathrm{R}}$.

Given these three pieces of information, we may be able to chart the atomic composition of baseline and altered human consciousness in a common DID space (an example of this is shown in Figure 5).

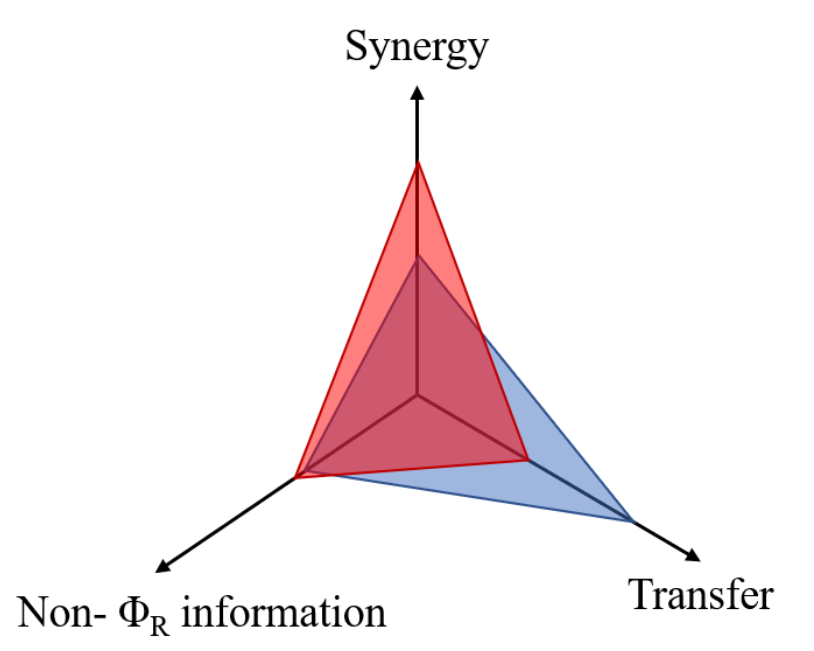

Figure 5. A low-dimensional representation of the information-theoretic atomic compositions of different systems. Each system (here, one shown in blue, the other in red) has a value for each of the three axes (which here summarise the full atomic composition) Synergy-containing atoms and information transfer are the two constituent elements of $\Phi_{R}$. The sum of a system's projections on each axis represents its total information flow (TDMI).

The atomic composition of $\Phi_{\mathrm{R}}$ may be found empirically by identifying an appropriate level at which neural information operates, and then quantifying the prevalence of each atom in the human brain at baseline and during altered states of consciousness. While this endeavour is far from straightforward, there is at least a plausible understanding of how we may address this challenge. Importantly, while current neuroimaging techniques might not be able to provide a description at the desirable level of resolution, it is still possible for them to provide a trustworthy reflection of the lower level. Hence, the main challenge is finding out what each atom of $\Phi_{R}$ is like, i.e. find a phenomenological answer to "what it is like to be a bit". The combination of ФID with phenomenological interpretations of information-theoretic atoms is therefore the core of our proposed framework, IID. 
Here we propose different (but potentially complementary) strategies that could be followed to identify which modes of consciousness could correspond to each of the atoms of $\Phi_{\mathrm{R}}$. One avenue is to consider altered states of consciousness that one may expect to show an abundance - or lack of - specific atoms, and understand how these differences might be reflected in the corresponding phenomenological alterations. These differences may be due to alterations induced by drugs (e.g., psychedelics, anaesthesia), neurological conditions, or even regular physiological alterations such as sleep, dreaming or meditation. For instance, deep meditation and psychedelics such as LSD can produce a state characterized by "loss of self", or "ego dissolution", and we may seek to identify which $\Phi I D$ atoms or combination thereof correspond to this phenomenology by examining the information decomposition of neural signals during such states. Because this approach takes phenomenological evidence as a starting point, we refer to it as the "a posteriori" $\Psi$ ID approach.

An alternative approach to relate phenomenology and $\Phi I D$ atoms is to try to identify what each atom may correspond to, in terms of subjective experience, based on what the atoms capture in terms of information processing. This may lead to predictions about how phenomenology should change if the atom in question were manipulated. Such predictions may then be tested empirically by studying perturbations that have been shown to alter the atomic composition (which may be discovered through the first (a posteriori) avenue described above). Because it starts from abstract considerations in terms of information processing, we refer to this avenue as the "a priori" approach of $\Psi$ ID.

\section{Empirical predictions: $\Phi$ nomenology}

In the following sections, we outline how the theoretical framework of $\Psi$ ID proposed here could function in practice, by conjecturing specific equivalences between $\Phi$ ID atoms and various aspects of phenomenology and other psychological phenomena. These provisional predictions are empirically testable using current neuroimaging techniques, and illustrate the practical value of $\Psi$ ID.

560 At this point, it is worth recapitulating the extent and structure of our theoretical commitments.

561 A fundamental assumption of this work is that it is theoretically possible and meaningful to quantify subjective experience (consciousness) in terms of integrated information of some (suitably chosen) neural dynamics. Subsequent to this background assumption, the core of our contribution is the hypothesis that it may be possible to obtain further insight into subjective experiences in terms of individual information-theoretic atoms, based on the $\Phi I D$ framework.

\section{Me, Myself, and $\Phi$ : Self and Persistent Synergies}

567 A fundamental aspect of phenomenology that may be understood in terms of $\Psi$ ID is selfhood. Specifically, we propose that a system may constitute an individuated "self" to the extent that its dynamics allow a persistent macroscale description of the system that is causally decoupled (as described in the Section A deepness in the $\Phi$ : Quantifying causal emergence)) from its individual constituent parts - here understood as the microscale. In other words, we posit that a self is associated with neural dynamics in which the macroscale has a causal influence on its own dynamics, beyond what is explainable from the individual microscale components. As discussed in A deepness in the $\Phi$ : Quantifying causal emergence, this notion corresponds to the presence of persistent synergies in the system, i.e. the $\Phi$ ID atom of causal decoupling 
(Figures 2 and 3). Note that these underlying intuitions are aligned with those of other recent works that conceptualise selfhood in information-theoretic terms (Chang et al., 2020; Krakauer et al., 2020), although these works use mathematical frameworks that do not enjoy the expressive richness of IID (indeed, extension of these works with the ФID framework would be an exciting avenue for future work).

Seen from this perspective, Hume's account of a "bundle of perceptions" (Treatise of Human Nature, Sect. VI. Of Personality, 1739/1986) ignores one fundamental issue: namely, that the parts together can constitute a whole beyond their sum, and high-order structures can persist even when the parts change - as has been formally demonstrated through ФID (Rosas et al., 2020). Thus, our proposition mirrors theories such as IIT, which take the unity of experience as their starting point and explain consciousness in terms of integration of information. Indeed, selfhood and consciousness seem tightly bound together in everyday experience. However, we know that they can be dissociated. Examples of a dissociation between consciousness and sense of self are experienced meditators and people under the influence of psychedelics: in both cases, people often report feeling a reduction in their experienced sense of self without acknowledging a diminution of their overall subjective experience - in fact, quite the opposite (see Deane et al., 2020). On the other hand, the effect of general anaesthesia on sense of self is also a topic of recent interest (Sleigh et al., 2020).

At this point, it is worth distinguishing the notions of selfhood from sense of self. The former concerns the extent to which a system can be demarcated from its environment and stably exist as an independent entity - the extent to which that system constitutes an individual (Weber \& Varela 2002; Thompson 2007). Following Krakauer et.al. (2020), we take a system's selfhood to be a graded property, which is determined by the system's dynamics (Levin 2019). Accordingly, selfhood pertains to systems at all levels of organisation, from humans to paramecia. In contrast, by sense of self we refer to the extent to which an organism perceives itself as having or being a self, which is related to higher-level metacognition (but see Friston (2018) for an alternative suggestion). Therefore, a sense of self requires some degree of selfhood that can be acknowledged by the organism's (possibly inaccurate) read-out of their own selfhood, whereas it is possible to have selfhood without a sense of self if the corresponding metacognitive abilities are not available. We leave open the question of whether any systems may, despite lacking selfhood, nevertheless come to have a delusional belief in their own selfhood - that is, having a sense of self without selfhood. Though seemingly unlikely and even paradoxical, our framework does not rule this out from a formal standpoint - and indeed, surprising and striking dissociations between belief and reality can be observed e.g. in patients suffering from anosognosia, who can adamantly deny obvious deficits from which they suffer.

612 Preliminary empirical support for a relationship between selfhood and causal emergence (i.e. 613 persistent synergy in the system) is found in recent work that suggests the brain's default mode network to be highly synergistic (Luppi et al., 2020a). This association is promising, as the default mode network has been consistently implicated in self-referential processing (Qiu \& Northoff 2011) as well as the sense of self or "ego" (Carhart-Harris \& Friston 2010), and its 617 integrity is compromised during LSD-induced "ego dissolution" (Nour et al., 2016). 618 Furthermore, this network is also implicated in loss of consciousness, whether induced by 619 anaesthesia or severe brain injury (Luppi et al 2019; Vanhaudenhuise et al., 2010; Boveroux et 620 al., 2010; DiPerri et al. 2017; Luppi et al., 2020b). Thus, the DMN may constitute a nexus for 621 the confluence of synergy, consciousness and selfhood in the human brain. 
An important implication of identifying selfhood with the $\Phi I D$ atoms that correspond to causal decoupling, is that all systems that have selfhood would also be conscious - since causal decoupling atoms are among the consciousness-related atoms that compose $\Phi_{R}$. However, and perhaps surprisingly, the converse is not the case: transfer-only systems may be conscious despite having no selfhood, according to our proposed framework (see Figure 6). This observation highlights the information-theoretic source of another kind of objection levelled against IIT, based on an intuitive resistance to the notion of certain systems (namely, transferonly systems) being conscious. In effect, most of the counter-examples of synthetic systems with high $\Phi$ and arguably no consciousness display only transfer and no synergy (Oizumi et al., 2014). Fortunately, decomposing $\Phi$ into its $\Phi I D$ atoms allow us to refine the usage of this metric, giving the practitioner the power to decide whether transfer should be considered part of $\Phi$ (much like the subtraction of redundancy was excluded from $\Phi_{R}$ based on theoretical considerations about a metric of consciousness being non-negative). Thus, rather than using counterintuitive examples to reject the theory as a whole, we argue for refining the theory without throwing the baby out with the bathwater, by capitalising on the finer-grained understanding made possible by $\Phi I D$. 


\section{Zones of Thought}

652 One intriguing implication of the YID framework is that different environments may be conducive to the emergence of alternative modes of consciousness - just as they may be differently conducive to various forms of life. Most organisms harvest information about their environment through multiple sensory modalities. The various senses of these organisms often provide information that is, at least partly, about the same external stimuli. For example, the same event is often the cause of stimuli delivered by more than one modality, such as someone's words being conveyed by their lip movements as well as their speech. This suggests that an environment where events tend to provide complementary information across sensory modalities, can favour the presence of integration via synergy, and organisms that are able to exploit this property will likely find themselves at an advantage.

662

As an example, in a forest a red, but not green round fruit is edible, but only when it smells sweet, so that edible exemplars are identifiable only when the two senses are combined. Such an environment may be expected to favour informational synergy between smell and sight in animals that rely on this fruit for nourishment. Conversely, animals whose main source of sustenance is easily identified by a single property (e.g. a specific color or smell) may not benefit from synergy to the same degree. Thus, the neural architectures of different organisms may rely on different combinations of information-theoretic atoms, possibly shaped by their environment and evolutionary history - as suggested by recent empirical evidence demonstrating that human brains rely on synergy to a greater extent than macaque brains (Luppi et.al. 2020a).

If, as we have argued, selfhood corresponds to causal decoupling (i.e. persistent synergies in the system), then environments that favour or discourage synergy may have an effect on selfhood (and presumably also sense of self). This prediction could be tested by means of virtual reality devices, which could be used to provide participants with visual, auditory, and tactile stimuli whose contents are entirely unrelated (i.e. stimuli that provide no synergy), and subsequently probe whether alterations have occurred in their sense of self, and also in the prevalence of $\Phi I D$ atoms corresponding to causal decoupling in their neural dynamics, as measured e.g. with functional MRI or other neuroimaging modality. Intriguingly, a multi-user VR platform was recently found to produce 'ego dissolution' type experiences not incomparable to those produced by moderate doses of psychedelics (Glowacki et al., 2020).

682 Furthermore, as synergy derives from the advantage of combining different sensory sources, 683 our theory predicts a degradation of selfhood as the unifying factor (and, presumably, the 684 organism's perception of its own selfhood, i.e. its sense of self) when there is a reduced benefit of integration between different sources of sensory information. In addition to modifying the structure of the environment, as proposed above, another way to reach a similar condition would be to add independent noise in each sensory modality. Doing so would result in a reduction of signal-to-noise ratio and, hence, a reduction in the proportion of information that each sensory source can contribute towards synergy. Intriguingly, such a reduction in signalto-noise ratio may be part of the mechanism by which LSD induces its well-known egodissolving effects - complementary of effects taking place in top-down activity (e.g., CarhartHarris \& Friston 2019). In effect, the 5-HT-2A agonistic properties of LSD lead to dysregulated spontaneous neuronal activity (Nutt et.al. 2020), which may be expected to weaken the 
694 contingency between neuronal firing and external stimuli, hence introducing noise in each

695 channel and reducing the ability of different sensory sources to provide synergistic information

696 - corresponding to reduced causal decoupling, our proposed information-theoretic substrate for

697 selfhood.

698 The converse of these hypotheses suggests that providing stimulation across different sensory 699 modalities that allows for high synergy may reduce the extent of psychedelic-induced ego700 dissolution. Partial support to this hypothesis is provided by recent studies on the effect of 701 different stimuli under LSD, which suggest a competition between the psychedelic effect of 702 the drug and stimulus (Mediano et al., 2020). We predict that by increasing the synergy between 703 different sensory sources through concurrent stimulation of multiple sensory modalities, one 704 might reinforce the self-other boundary - and the subjective evaluation of it - resulting in 705 diminished ego-dissolution.

706

\section{Discussion}

708 We have provided theoretical contributions about the nature of consciousness (understood as 709 integrated information) based on its decomposition into elementary information-theoretic 710 atoms, and the key insight that the same total integrated information may be comprised of 711 different combinations of information atoms. This approach has allowed us to provide 712 empirical predictions that could be tested with the tools of contemporary neuroscience. A 713 schematic summary of our contributions, and the underlying assumptions, is provided in Figure 7147. 


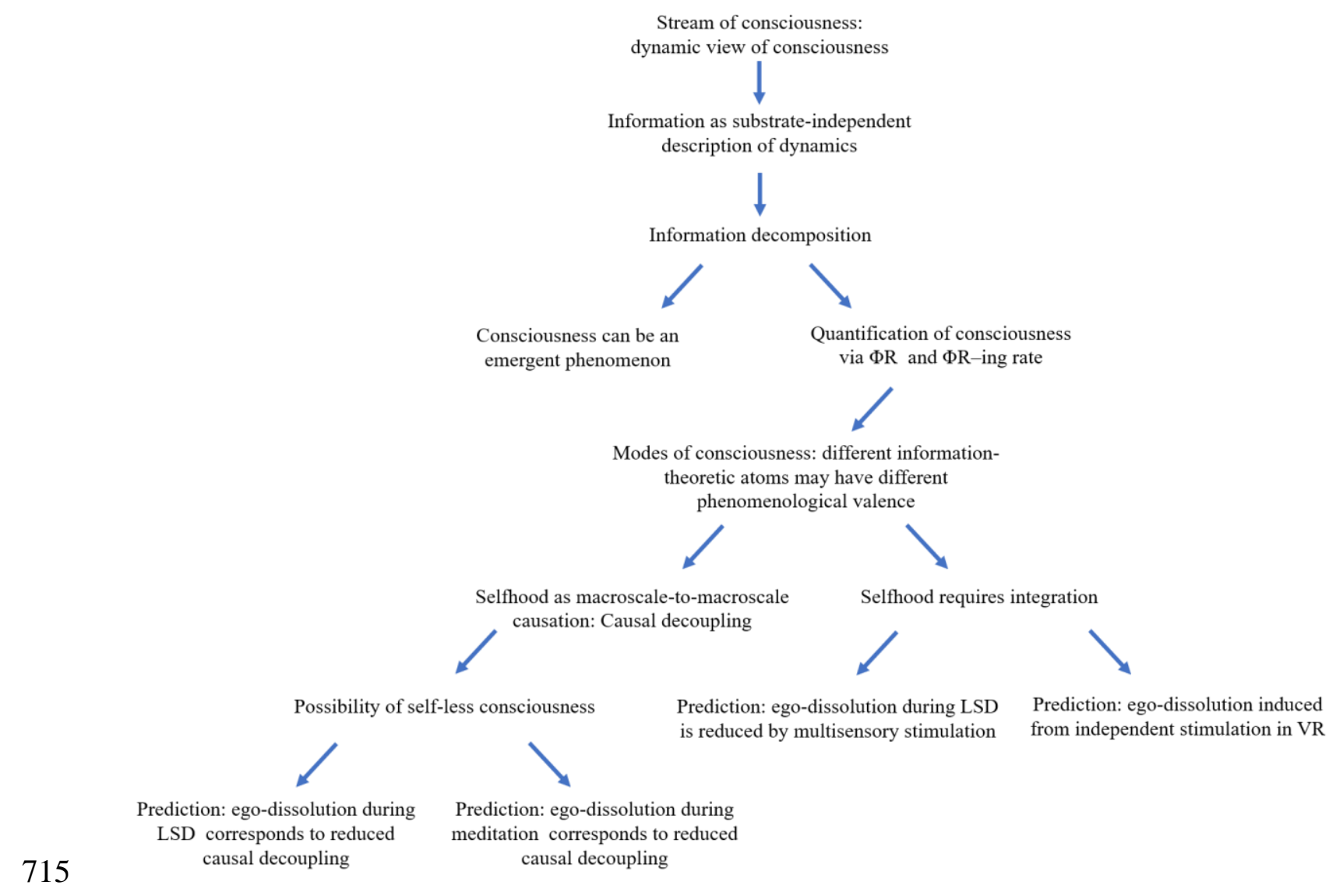

716 Figure 7. Visual summary of $\Psi$ ID, illustrating the logical structure between the various claims made in 717 this paper.

\section{To in $\Phi$ nity and beyond}

720 The main premise of this piece - and of $\Psi$ ID more broadly - is that there is merit to the principle of quantifying consciousness via integrated information (suitably defined). We acknowledge that the predictions of YID outlined here are broad and high-level (Figure 7); however, we believe that it is important for a theoretical account to be clear about its empirical commitments. Indeed, we have made predictions in a broad range of areas - many of which could already be tested. In fact, while there is still work to be done on finding the right temporal and spatial scale at which neural information should be best assessed, it is already possible to approach this quantification with effective results (see e.g. Luppi et al., 2020b).

Importantly, the advantages of a $\Phi I D-b a s e d$ account of consciousness are not restricted to IIT, but rather this framework represents a general lens through which to understand consciousness. Indeed, the presented framework also provides insights about other theoretical approaches to consciousness, such as the Global Neuronal Workspace Theory (Dehaene \& Changeux 2011; Mashour et al., 2020). In effect, by re-thinking information exchange in the human brain in terms of synergy and redundancy, it is possible to delineate a "synergistic global workspace" purely from functional considerations - based on brain regions whose connections are predominantly synergistic (Luppi et al 2020b). Furthermore, analysis of resting-state functional MRI data showed that loss of consciousness due to anaesthesia or brain injury corresponds to 
a reduction of $\Phi_{R}$ between regions of the synergistic workspace. In this way, the more nuanced view on neural information processes offered by $\Phi I D$ brings us closer to reconciling IIT's and GNWT's accounts of consciousness, and offers the promise of further insights for our understanding of consciousness.

741

742

743

744

Another intriguing future research direction is to explore the relationship between the elaboration of YID explored herein and hierarchical predictive coding and the Free Energy Principle (FEP) (see Seth \& Hohwy 2020). Interestingly, ideas in this direction have been put forward by Friston and colleagues in the context of IIT (Friston et al., 2020), where they suggest that - at least in terms of numerical analyses - the minimization of variational free-energy (which, according to the FEP, is a basic imperative for any self-maintaining cognitive system) maximizes $\Phi$ and vice versa (Friston et al., 2020). Thus, an exciting question for future research is to assess how the notion of free-energy minimising agents that also maximise $\Phi$, relates to our theoretical contributions put forward above: does maximisation of $\Phi$ also correspond to maximisation of $\Phi_{R}$, and how is it reflected in the $\Phi_{R}$-ing rate? Is it driven by any specific $\Phi I D$ atoms - such as synergy or transfer? Additionally, one could ask about the relationship between downward causation, discussed throughout our paper, to predictive mechanisms in a control hierarchy; specifically, where a higher-level component predicts some general feature of lowerlevel constituents. We thus believe there are promising overlaps between our account of YID and the theoretical ambitions of predictive coding and FEP approaches, which deserve to be explored in the future.

\section{So long, and thanks for all the $\Phi$ s}

The distinction of different $\Phi I D$ atoms within integrated information leads to a new understanding of the space of possible consciousness, providing a phenomenological understanding of information-theoretic atoms: we call this framework YID. This framework provides not only a revised measure of integrated information, $\Phi_{\mathrm{R}}$, but also a way to measure the efficiency of a system at turning information into consciousness - i.e. its " $\Phi_{\mathrm{R}}$-ing rate". Importantly, when combined with a mathematical framework for the definition of causal emergence, YID enables us to propose a formal solution to the long-standing question of whether consciousness is an emergent phenomenon: namely, we show that both consciousness (understood as integrated information, $\Phi_{R}$ ) and emergence each comprise distinct sets of information-theoretic atoms, and only some of them overlap.

Thus, whether consciousness is emergent or not will depend on its information-theoretic composition. Moreover, since $\Phi_{\mathrm{R}}$ is composed of different atoms, different systems may achieve the same amount of integrated information through different combinations of information-theoretic atoms. By investigating the phenomenology associated with specific atoms, these "modes of consciousness" may provide a way to eventually understand the phenomenology of different states of consciousness that are not immediately accessible.

In addition to these theoretical contributions for a more nuanced understanding of

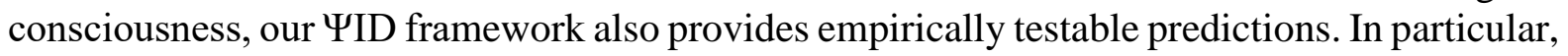
we have hypothesised that $\Phi I D$ atoms involving synergy may correspond to modes of being conscious that involve a sense of self. We look forward to empirical tests of these predictions, and the possible extension of our work to other mental phenomena, such as psychiatric conditions. 


\section{Acknowledgements}

781 The authors are grateful to Marta Halina and members of her research group for helpful discussions. The authors would also like to acknowledge the intellectual influence of Dr. Vernor Vinge, whose vision helped to shape the context of this work - especially as laid out in the Zones of Thought series: A Fire upon the Deep, and A Deepness in the Sky. The authors believe that the influence of works of science fiction in shaping scientific inquiry is insufficiently acknowledged in the scientific literature, and wish to contribute to redress this state of affairs.

This work was supported by the Gates Cambridge Trust (to AIL); the Stephen Erskine Fellowship (Queens' College, Cambridge, to EAS); the Wellcome Trust (grant no. 210920/Z/18/Z; to PAM and DB); The History and Philosophy of Science Trust Fund (to DJH); and the Ad Astra Chandaria foundation (to FR); Ad Astra Trust, Tim Ferriss, The Nikean Foundation, and The Tamas Family (to (RLC-H).

793

794

795

796

797

798

799

800

\section{Data availability}

Code to perform Integrated Information Decomposition is available upon request from author PAM: email pam83@cam.ac.uk.

\section{References}

Atasoy, S., Roseman, L., Kaelen, M., Kringelbach, M.L., Deco, G., \& Carhart-Harris, R.L. 2017. Connectome-harmonic decomposition of human brain activity reveals dynamical repertoire re-organization under LSD. Scientific Reports, vol.7: 17661. https://doi.org/10.1038/s41598-017-17546-0

Atasoy, S., Vohryzek, J., Deco, G., Carhart-Harris, R.L., \& Kringelbach, M.L. 2018. Common neural signatures of psychedelics: Frequency-specific energy changes and repertoire expansion revealed using connectome-harmonic decomposition. Progress in Brain Research, vol. 242: 97-120. https://doi.org/10.1016/bs.pbr.2018.08.009

Balduzzi, D. \& Tononi, G. 2008. Integrated Information in Discrete Dynamical Systems: Motivation and Theoretical Framework. PLoS Computational Biology, doi: https://doi.org/10.1371/journal.pcbi.1000091 
808

809

810

811

812

813

814

815

816

817

818

819

820

821

822

823

824

825

826

827

828

829

Balduzzi, D. \& Tononi, G. 2009. Qualia: The Geometry of Integrated Information. PLoS Computational Biology, vol. 5(8): e1000462. https://doi.org/10.1371/journal.pcbi.1000462

Barrett, A.B. 2014. An integration of integrated information theory with fundamental physics. Frontiers in Psychology, vol.5 (63). DOI: https://doi.org/10.3389/fpsyg.2014.00063

Barrett, A.B. \& Seth A.K. 2011. Practical measures of integrated information for time series data. PLoS Computational Biology, vol.7, e1001052. DOI: 10.1371/journal.pcbi.1001052

Barttfeld, P., Uhrig, L., Stitt, J., Sigman, M., Jarraya, B., and Dehaene, S. 2015. Signature of consciousness in the dynamics of resting-state brain activity. Proceedings of the National Academy of Sciences, vol. 112(3): 887-892. doi.org/10.1073/pnas.1418031112

Bedau, M.A. 1997. Weak emergence. Philosophical perspectives, vol.11: 375-399. DOI: $\underline{10.1111 / 0029-4624.31 . s 11.17}$

Bedau, M.A., 2002. Downward causation and the autonomy of weak emergence. Principia: An international journal of epistemology, vol.6(1): 5-50. DOI: $\underline{10.7551 / \mathrm{mitpress} / 9780262026215.003 .0010}$

Bertschinger, N., Rauh, J., Olbrich, E., Jost, J. \& Ay, N. 2014. "Quantifying Unique Information.” Entropy, vol. 16 (4): 2161-2183. DOI: https://doi.org/10.3390/e16042161

Boveroux, P., Vanhaudenhuyse, A., Bruno, M.A., Noirhomme, Q., Lauwick, S., Luxen, A., Degueldre, C., Plenevaux, A., Schnakers, C., Phillops, C., Brichant, J.F., Bonhomme, V., Maquet, C., Greicius, M.D., Laureys, S., \& Boly, M. 2010. Breakdown of withinand between-network rsting stake functional magnetic resonance imaging connectivity 
Breakspear, M., Williams, L.M. \& Stam, C.J. 2004. A novel method for the topographic analysis of neural activity reveals formation and dissolution of dynamic cell assemblies. Journal of Computational Neurosceicne, vol.16(1): 49-69. DOI: https://doi.org/10.1023/B:JCNS.0000004841.66897.7d

836 Cai, L., Wang, J., Guo, Y., Lu, M., Dong, Y., Wei, X. 2020. Altered inter-frequency dynamics of brain networks in disorders of consciousness. Journal of Neural Engineering, vol.

Cao, Y., Summerfield, C., Park, H., Giordana, B.L., \& Kayser, C. 2019. Causal Inference in the Multisensory Brain. Neuron, vol.102: 1076-1087. DOI: https://doi.org/10.1016/j.neuron.2019.03.043

Carhart-Harris, R.L. \& Friston, K. 2010. The default-mode, ego-functions and free-energy: a neurobiological account of Freudian ideas. Brain: A Journal of Neurology, vol.133(4): 1265-1283. DOI: https://doi.org/10.1093/brain/awq010

Carhart-Harris, R.L. \& Friston, K. 2019. REBUS and the Anarchic Brain: Toward a Unified Model of the Brain Action of Psychedelics. Pharmacological Reviews, vol.71(3): 316-

Casali, A.G., Gosseries, O., Rosanova, M., Boly, M., Sarasso, S., Casali, K.R., Casarotto, S., 849 Bruno, M.A., Laureys, S., Tononi, G., \& Massimini, M.A. 2013. Theoretically Based 344. DOI: https://doi.org/10.1124/pr.118.017160 
852 Cavanna, F.,Vilas, M.G., Palmucci, M. \& Tagliazucchi, E. 2018. Dynamic Functional 853 Connectivity and Brain Metastability during Altered States of Consciousness. 854 NeuroImage, vol. 180: 383-395. DOI: http://doi.org/10.1016/j.neuroimage.2017.09.065.

855 Cea, I. 2020. Integrated Information Theory of Consciousness is a Functionalist Emergentism.

856 Synthese, DOI: https://doi.org/10.1007/s11229-020-02878-8

857 Chang, A.Y.C., Biehl, M., Yu, Y. \& Kanai, R. 2020. Information Closure Theory of 858 Consciousness.

Frontiers

in Psychology,

DOI:

859 https://doi.org/10.3389/fpsyg.2020.01504

860

861

Colombetto, G. \& Ratcliffe, M. 2012. Bodily Feeling in Depersonalization: A 862 Phenomenological Account. Emotion Review, $\operatorname{vol.4(2):~}$

Crick, F. \& Koch, C. 2003. A framework for consciousness. Nature Neuroscience, vol.6: 119864 126. DOI: https://doi.org/10.1038/nn0203-119

Deane, G. 2020. Dissolving the self: Active inference, psychedelics, and ego-dissolution. 866 Philosophy and the Mind Sciences, vol. 1(1): https://doi.org/10.33735/phimisci.2020.I.39

Deane, G., Miller, M. \& Wilkinson, S. 2020. Losing Ourselves: Active Inference, Depersonalization, and Meditation. Frontiers in Psychology, 11:539726. Doi: https://doi.org/10.3389/fpsyg.2020.539726

De Caro, M. \& Grasso, M. 2017. Three Views on Mental Downward Causation. In Paoletti, M.P. \& Orilla, F. Philosophical and Scientific Perspectives on Downward Causation, New York: Routledge. 
874 Deco, G., Cruzat, J., Cabral, J., Tagliazucchi, E., Laufs, H., Logothetis, N.K., \& Kringelbach, 875 M.L. 2019. Awakening: Predicting external stimulation to force transitions between 876 different brain states. Proceedings of the National Academy of Sciences, vol. 116(36): 18088-18097. https://doi.org/10.1073/pnas.1905534116

878

879

880

881

882

883

884

885

886

887

888

889

890

891

892

893

894

895

Dehaene, S. \& Changeuz, J.P. 2011. Experimental and Theoretical Approaches to Conscious $\begin{array}{llll}\text { Processing. } & \text { Neuron, } & \text { vol. } & \text { 200-227. }\end{array}$ https://doi.org/10.1016/j.neuron.2011.03.018

Demertzi, A., Tagliazucchi, E., Dehaene, S. .. \& \& Stitt, J.D. 2019. Human consciousness is supported by dynamic complex patterns of brain signal coordination. Science Advances Research Article, vol. 5: eeat7603. DOI: $\underline{10.1126 / \text { sciadv.aat7603 }}$

Dinesh, P., Li, D., Dean, J.G., Brito, M., Liu, T., Fryzel, A.M., Hudetz, A.G., \& Mashour, G.A. 2020. Level of Consciousness is Dissociable from Electroencephalographic Measures of Cortical Connectivity, Slow Oscillations, and Complexity. Journal of Neuroscience, vol.40(3): 605-618. DOI: https://doi.org/10.1523/JNEUROSCI.1910-19.2019

Di Perri, C., Amico, E., Heine, L., Annen, J., Martial, C., Larroque, S.K., Soddu, A., Marinazzo, D., \& Laureys, S. 2017. Multifaceted Brain Networks Reconfiguration in Disorders of Consciousness Uncovered by Co-Activation Patterns. Human Brain Mapping, vol.39(1): 89-103. DOI: https://doi.org/10.1002/hbm.23826

Eagelman, S., Chander, D., Reynolds, C., Ouellette, N.T., \& MacIver, M.B. 2019. Nonlinear dynamics captures brain states at different levels of consciousness in patients anesthetized with propofol. PLOS One, vol. 14(10): e0223921. https://doi.org/10.1371/journal.pone.0223921 
896 Feder, M. \& Merhav, N. 1994. Relations between entropy and error probability. IEEE 897 Transactions on Information Theory, vol.40(1): 259-266. DOI: 10.1109/18.272494

898

899

900

901

902

903

904

905

906

907

908

909

910

911

912

913

914

915

916

Feinberg, T.E. \& Mallot, J. 2020. Phenomenal Consciousness and Emergence: Eliminating the Explanatory Gap. Frontiers in Psychology, 11:1041. Doi: https://doi.org/10.3389/fpsyg.2020.01041

Friston, K. 2012. A Free Energy Principle for Biological Systems. Entropy, vol. 14(11): 21002121. DOI: $\underline{\text { https://doi.org/10.3390/e14112100 }}$

Friston, K. 2018. Am I Self-Conscious? (Or Does Self-Organization Entail SelfConsciousness?). Frontiers in Psychology, vol.9. DOI: https://doi.org/10.3389/fpsyg.2018.00579

Friston, K., Sengupta, B. \& Auletta, G. 2014. Cognitive Dynamics: From Attractors to Active Inference. Proceedings of the IEEE, vol. 102 (4): 427-445., doi: https://doi.org/10.1109/jproc.2014.2306251

Friston, K.J., Wiese, W., \& Hobson, A.J. 2020. Sentience and the Origins of Consciousness: From Cartesian Duality to Markovian Monism. Entropy, vol.22(5), 516. DOI: https://doi.org/10.3390/e22050516

Glowacki, D.R., Wonnacott, M.D., Freire, R., Glowacki, B.R., Gale, E.M., Pike, J.E., de Haan, T., Chatziapostolou, M. \& Metlata, O. 2020. Isness: Using Multi-Person VR to Design Peak Mystical Type Experiences Comparable to Psychedelics. CHI 20: Proceedings of the 2020 CHI Conference on Human Factors in Computing Systems, 1-14. DOI: https://doi.org/10.1145/3313831.3376649 
917 Griffith, V., \& Koch, C. 2014. “Quantifying Synergistic Mutual Information.” Guided Self-

918 Organization: Inception Emergence, Complexity and Computation: 159-190. doi: $919 \quad$ https://doi.org/10.1007/978-3-642-53734-9_6

920 Hain,G., Zamora-Lopez, G., Uhrig, L., Tagliazucchi, E., Laufs, H., Mantini, D., Kringelbach, 921 M., Jarraya, B., Deco, G. 2021. Signature of consciousness in brain-wide 922 923 synchronization patterns of monkey and human fMRI signals. NeuroImage, vol. 226:

Hellman, M. \& Raviv, J. 1970. Probability of error, equivocation, and the Chernoff bound. IEEE Transactions on Information Theory, vol.16(4): 368-372. DOI: https://doi.org/10.1109/TIT.1970.1054466

Hesp, C., Smith, R., Parr, T., Allen, M., Friston, K. \& Ramstead, M. 2019. Deeply Felt Affect:

929 The Emergence of Valence in Deep Active Inference. PsyArXic Preprints, DOI:

930 $\underline{10.31234 / \text { osf.io/62pfd }}$

Huang, Z., Zhang, J., Wu, J., Mashour, G.A., Hudetz, A.G. 2020. Temporal circuit of 932 macroscale dynamic brain activity supports human consciousness. Science Advances, vol.6(11): eaaz0087. DOI: 10.1126/sciadv.aaz0087

Hume, D. 1986. A Treatise of Human Nature: Being an Attempt to Introduce the Experimental 935 Method of Reasoning. London: Penguin Classics.

Hutcinson, R.M., Hutchinson, M., Manning, K.Y., Menon, R.S., \& Everling S. 2014. Isolurane 937 induces dose-dependent alterations in the cortical connectivity profiles and dynamic properties of the brain's functional architecture. Human Brain Mapping, vol.35(12): 
939

940

\section{1}

942

943

944

945

946

947

948

949

950

951

952

953

954

955

956

957

958

959

5754-75.

doi:10.1002/hbm.22583.

James R.G., Ellison, C., \& Crutchfield, J. 2011. Anatomy of a bit: Information in a time series observation. Chaos: An Interdisciplinary Journal of Nonlinear Science, vol. 21(3). https://doi.org/10.1063/1.3637494

James, R.G. \& Crutchfield, J.P. 2017. Multivariate Dependence Beyond Shannon Information. Entropy, vol.19(10), 531. https://doi.org/10.3390/e19100531

Jaynes, T. 2003. Probability Theory: The Logic of Science. Cambridge: Cambridge University Press.

Kleiner, J. 2020. Mathematical Models of Consciousness. Entropy, 22(6). DOI: https://doi.org/10.3390/e22060609

Krakauer, D., Bertschinger, N., Olbrich, E., Flack, J.C. \& Ay, N. 2020. The information theory of individuality. Theory in Biosciences, vol.139: 209-223. DOI: https://doi.org/10.1007/s12064-020-00313-7

Lee., H., Golkowski, D., Jordan, D., Berger, S., Ilg, R., Lee, J. Mashour, G.A., \& Lee, U. 2019. Relationship of critical dynamics, functional connectivity, and states of consciousness in large-scale human brain networks. Neuroimage, vol.188: 228-238. DOI: https://doi.org/10.1016/j.neuroimage.2018.12.011

Levin, M. 2019. The Computational Boundary of a "Self": Developmental Bioelectricity Drives Multicellularity and Scale-Free Cognition. Frontiers in Psychology, DOI: https://doi.org/10.3389/fpsyg.2019.02688 
Lord L.D., Expert, P., Atasoy, S., Roseman, L., Rapuano, K., Lambiotte, R., Nutt, D.J., Deco,

961

962

963

964

965

966

967

G., Carhart-Harris, R.L., Kringelbach, M.L., Cabral, J. 2019. Dynamical exploration of the repertoire of brain networks at rest is modulated by psilocybin. NeuroImage, vol.199: 127-142. DOI: $\underline{\text { https://doi.org/10.1016/j.neuroimage.2019.05.060 }}$

Liuzzi, L., Gascoyne, L.E., Tewarie, P.K., Barratt, E.L., Boto, E., \& Brookes, M.J. 2017. Optimising Experimental Design for MEG Resting State Functional Connectivity Measurement. $\quad$ NeuroImage, $\quad$ vol.155: 565-576. DOI: https://doi.org/10.1016/j.neuroimage.2016.11.064.

Lizier, J., Bertschinger, N., Jost, J., \& Wibral, M. 2018. “Information Decomposition of Target Effects from Multi-Source Interactions: Perspectives on Previous, Current and Future Work." Entropy, vol. 20(4): 307. https://doi.org/10.3390/e20040307

Luppi, A., Craig, M., Pappas, I., Finoia, P., Williams, G.B., Allanson, J., Pickard, J.D., Owen, A.M., Naci, L., Menon, D.K., \& Stamatakis, E.A. 2019. Consciousness-specific dynamic interactions of brain integration and functional diversity. Nature Communications, vol.10: 1-12. https://doi.org/10.1038/s41467-019-12658-9

Luppi, A., Mediano, P.A.M., Rosas, F.E., Holland, N., Fryer, T.D., O’Brien, J.T., Rowe, J.B., Menon, D.K., Bor, D., \& Stamatakis, E.A. 2020a. A synergistic core for human brain evolution and cognition. bioRxiv preprints, DOI: https://doi.org/10.1101/2020.09.22.308981

Luppi, A., Mediano, P.A.M., Rosas, F.E., Allanson, J., Pickard, J.D., Carhart-Harris, R.L., Williams, G.B., Craig, M.M., Finoia, P., Owen, A.M., Naci, L., Menon, D.K., Bor, D., \& Stamatakis, E.A. 2020b. A Synergistic Workspace for Human Consciousness 

https://doi.org/10.1101/2020.11.25.398081

984

985

986

987

988

989

990

991

992

993

994

995

996

997

998

999

1000

1001

1002

1003

Luppi, A., Vohryzek, J., Kringelbach, M.L., Mediano, P.A.M., Craig, M.M., Adapa, R., Carhart-Harris, R.L., Roseman, L., Pappas, I., Finoia, P., Williams, G.B., Allanson, J., Pickard, J.D., Menon, D.K., Atasoy, S., \& Stamatakis, E.A 2020c. Connectome Harmonic Decomposition of Human Brain Dynamics Reveals a Landscape of Consciousness.

bioRxiv: https://doi.org/10.1101/2020.08.10.244459

Luppi, A., Carhart-Harris, R., Roseman, L., Pappas, I., Menon, D.K., \& Stamatakis, E.A. 2020d. LSD alters dynamic integration and segregation in the human brain. Neuroimage, vol. 227, doi: https://doi.org/10.1016/j.neuroimage.2020.117653

Mashour, G., Roelfsema, P., Changeux, J.P., \& Dehaene, S. 2020. Conscious Processing and the Global Neuronal Workspace Hypothesis. Neuron, vol. 105(5): 776-798. DOI: $\underline{10.1016 / j . n e u r o n .2020 .01 .026}$

Mediano, P., Seth, A.K., \& Barrrett, A.B. 2018. Measuring Integrated Information: Comparison of Candidate Measures in Theory and Simulation. Entropy, vol.21(1), 17. DOI: https://doi.org/10.3390/e21010017

Mediano, P., Rosas, F., Carhart-Harris, R.L., Seth, A.K., \& Barrett, A. 2019. Beyond integrated information: A taxonomy of information dynamics phenomena. Neurons and Cognition. Available from arXiv:1909.02297

Mediano, P.A.M., Rosas, F.E., Timmerman, C., Roseman, L., Nutt, D.J., Fielding, A., Kaelen, M., Kringelbach, M.L., Barrett, A.B., Seth, A.K., Muthukumaraswamy, S., Bor, D. \& 

neurodynamics. bioRxiv preprints, DOI: https://doi.org/10.1101/2020.11.01.356071

1006

1007

1008

1009

1010

1011

1012

1013

1014

1015

1016

1017

1018

1019

1020

1021

1022

1023

Moon, K. \& Pae, H. 2019. Making Sense of Consciousness as Integrated Information Evolution and Issues of Integrated Information Theory. The Journal of Cognitive Psychology, vol. 20(1): 1-52.

Nagel, T. 1974. What Is It Like to Be a Bat? The Philosophical Review, vol.83(4): 435-450. https://doi.org/10.2307/2183914

Northoff, G., Wainio-Theberge, S. \& Evers, K. 2020. Is Temporo-Spatial Dynamics the ‘Common Currency’ of Brain and Mind? In Quest of ‘Spatiotemporal Neuroscience. Physics of Life Reviews, vol. 33: 34-54. DOI: https://doi.org/10.1016/j.plrev.2019.05.002

Nour, M.M., Evans, L., Nutt, D., \& Carhart-Harris, R.L. 2016. Ego-Dissolution and Psychedelics: Validation of the Ego-Dissolution Inventory (EDI). Frontiers in Human Neuroscience, DOI: https://doi.org/10.3389/fnhum.2016.00269

Nutt, D., Erritzoe, D. \& Carhart-Harris, R.L. 2020. Psychedelic Psychiatry's Brave New World. Cell Commentary, vol.181(1): 24-28. DOI: https://doi.org/10.1016/j.cell.2020.03.020

O'neill, G.C., Tewarie, P., Vidaurre, D., Liuzzi, L., Wollrich, M.W., Brookes, M.J. 2018. Dynamics of Large-Scale Electrophysiological Networks: A Technical Review. NeuroImage, vol.180: 559-576. DOI: https://doi.org/10.1016/j.neuroimage.2017.10.003 
1024 Oizumi, M., Albantakis, L., Tononi, G. 2014. From the Phenomenology to the Mechanisms of 1025 Consciousness: Integrated Information Theory 3.0. PLoS Computational Biology, vol.10(5). DOI: https://doi.org/10.1371/journal.pcbi.1003588

1027 Oizumi, M., Tsuchiya, N. \& Amari, S. 2016. Unified Framework for Information Integration 1028 Based on Information Geometry. Proceedings of the National Academy of Sciences, vol. 113(51): 14817-14822. DOI: https://doi.org/10.1073/pnas.1603583113.

1030 Palacios, E.R., Razi, A., Parr, T., Kirchhoff, M., \& Friston, K. 2017. Biological SelfOrganisation and Markov Blankets. bioRxiv, doi: https://doi.org/10.1101/227181

Palacios, E.R., Isomura, T., Parr, T. \& Friston, K. 2019. The Emergence of Synchrony in Networks of Mutually Inferring Neurons. Scientific Reports, vol. 9(1). DOI: https://doi.org/10.1038/s41598-019-42821-7

Parr, T. \& Friston, K. 2018. Generalised Free Energy and Active Inference: Can the Future Cause the Past? Biological Cybernetics, vol. 113: 495-513. DOI: https://doi.org/10.1007/s00422-019-00805-w

Parrondo, J.M.R., Horowitz, J.M., \& Sagawa, T. 2015. Thermodynamics of information. Nature Physics 11: 131-139. DOI: https://doi.org/10.1038/nphys3230

Rosas F., Ntranos, V., Ellison, C.J., Pollin, S., \& Verhelst, M. 2016. Understanding Interdependency Through Complex Information Sharing. Entropy, vol.18(2), 38:

Rosas, F.E., Mediano, Pedro, A.M., Jensen, J.H., Seth, A.K., Barrett, A.B., Carhart-Harris, R.L., Bor, D. 2020. Reconciling emergences: An information-theoretic approach to identify causal emergence in multivariate data. arXiv preprint: arXiv:2004.08220. 
1046

1047

1048

1049

1050

1051

1052

1053

1054

1055

1056

1057

1058

1059

1060

1061

1062

1063

1064

1065

Schwitzgebel, E. 2016. Phenomenal Consciousness, Defined and Defended as Innocently as I Can Imagine. Journal of Consciousness Studies, vol. 23(11-12): 224-235.

Searle, John R. (mills Professor Of Mind And Language, Univ. Mind - a Brief Introduction. Oxford University Press, 2005.

Seth, A. \& Friston, K. 2016. Active interoceptive inference and the emotional brain. Philosophical Transations of the Royal Society B, vol. 371(1708): https://doi.org/10.1098/rstb.2016.0007

Seth, A.K. \& Hohwy, J. 2020. Predictive processing as an empirical theory for consciousness science. Cognitive Neuroscience Preprints, DOI: 10.1080/17588928.2020.1838467

Shannon, C.E. 1948. A Mathematical Theory of Communication. The Bell System Technical Journal, $\quad$ vol.27: $\quad 379-423, \quad$ 623-656. $\quad$ DOI: $\quad$ https://doi.org/10.1002/j.1538$\underline{\text { 7305.1948.tb01338.x }}$

Sleigh, J., Warnaby, C. \& Tracey I. General Anaesthesia as fragmentation of selfhood: insights from electroencephalography and neuroimaging. British Journal Anaesthesia, vol. 121(1): 233-240. Doi: 10.1016/j.bja.2017.12.038

Standage, D., Areshenkoff, C.N., Nashed, J.Y., Hutchinson, R.M., Hutchinson, M., Heinke, D., Menin, R.S., Everling, S., \& Gallivan, J.P. 2020. Dynamic Reconfiguration, Fragmentation, and Integration of Whole-Brain Modular Structure across Depths of Consciousness. Cerebral Cortex, vol. 30(10): 5229-5241. https://doi.org/10.1093/cercor/bhaa085 
1066

1067

1068

1069

1070

1071

1072

1073

1074

1075

1076

1077

1078

1079

1080

1081

1082

1083

1084

1085

1086

1087

Stramaglia, S., Cortes, J.M., \& Marinazzo D. 2014. Synergy and redundancy in the Granger causal analysis of dynamical networks. New Journal of Physics, vol.16(10), 105003: arXiv:1403.5156

Tononi, G. 2004. An information theory of consciousness. BMC Neuroscience, vol.5, 42. DOI: https://doi.org/10.1186/1471-2202-5-42

Tononi, G. \& Koch, C. 2015. Consciousness: here, there, and everywhere? Philosophical Transactions of the Royal Society, $B$ 370: 20140167. DOI: https://doi.org/10.1098/rstb.2014.0167

Tononi, G., Boly, M., Massimini, M., \& Koch, C. 2016. Integrated Information Theory: from Consciousness to Its Physical Substrate. Nature Reviews Neuroscience, vol.17(7): 450461. DOI: https://doi.org/10.1038/nrn.2016.44

Tononi, G., Sporns, O. \& Edelman, G. 1994. A measure for brain complexity: relating functional segregation and integration in the nervous system. Proc. Natl. Acad. Sci. USA, vol.91(11): 5033-7. DOI: 10.1073/pnas.91.11.5033

Tononi, G., Sporns, O., \& Edelman, G. 1998. Complexity and coherency: integrating information in the brain. Trends in Cognitive Science, vol.2(12): 474-84. DOI: $\underline{10.1016 / \mathrm{s} 1364-6613(98) 01259-5}$

Tononi, G. \& Edelman, G. 1998. Consciousness and Complexity. Science, vol. 282(5395): 1856-1851. DOI: $10.1126 /$ science.282.5395.1846

Turkheimer, F.E., Rosas, F.E., Dipasquale, O., Martins, D., Fagerholm, E.D., Expert, P., Vasa, F., Lord, L.D., Leech, R. 2020. A Complex Systems Perspective on Neuroimaging Studies of Behaviour and Its Disorders. Complex Systems Perspective on Neuroimaging 

Studies of Behaviour and its Disorders Preprints. doi:10.20944/preprints202008.0654.v1.

Tsuchiya, N., Taguchi, S., Saigo, H. 2016. Using category theory to assess the relationship between consciousness and integrated information theory. Neuroscience Research, vol 107: 1-7. Doi: https://doi.org/10.1016/j.neures.2015.12.007

1093

Uhrig, L., Stitt, J.D., Jacob, A., Tasserie, J., Barttfeld, P., Dupont, M., Dehaene, S., Jarraya, B. 2018. Resting-state Dynamics as a Cortical Signature of Anaesthesia Monkeys. Anesthesiology, vol, 129(5): 942-958. DOI: 10.1097/ALN.0000000000002336

Wibral, M., Priesemann, V., Kay, J.W., Lizier, J.T., \& Philips, W.A. 2017. Partial Information Decomposition as a Unified Approach to the Characterization and Design of Neural Goal $\begin{array}{llll}\text { Functions. } & \text { BMC Neuroscience, } & \text { vol.16(S1). }\end{array}$ https://doi.org/10.1016/j.bandc.2015.09.004

Vanhaudenhuyse, A., Noirhomme, Q., Tshibanda, L.J.-F., Bruno, M.A., Boveroux, P., Schnakers, C., Soddu, A., Perlbarg, V., Ledoux, D., Brichant, J.-F., Moonen, G., Maquet, P., Greicius, M.D., Laureys, S., \& Boly, M. 2010. Default network connectivity reflects the level of consciousness in non-communicative brain-damaged patients. Brain: A Journal of Neurology, vol.133(1): 161-171. DOI: https://doi.org/10.1093/brain/awp313

Varly, T.F., Denny, V., Sporns, O., \& Patania, A. 2020a. Topological Analysis of Differential Effects of Ketamine and Propofol Anesthesia on Brain Dynamics. bioRxiv, doi: https://doi.org/10.1101/2020.04.04.025437

Varley, T.F., Luppi, A., Pappas, I., Naci, L., Adapa, R., Owen, A.M., Menon, D.K. \& Stamatakis, E.A. 2020b. Consciousness \& Brain Functional Complexity in Propofol 
1112 Vlisides, P.E., Li, D., Zierau, M., Lapointe, A.P., Ip, K.A., McKinney, A.M., \& Mashour, G.A.

1113 2019. Dynamic Cortical Connectivity during General Anesthesia in Surgical Patients.

1114 Anesthesiology, vol. 130: 885-897. https://doi.org/10.1097/ALN.0000000000002677

1115 Weber, A \& Varela, J. 2002. Life after Kant: Natural purposes and the autopoietic foundations

1116 of biological individuality. Phenomenology and the Cognitive Sciences, vol.1: 97-125.

1117 DOI: https://doi.org/10.1023/A:1020368120174

1118 Williams, P.L. 2011. Information Dynamics: Its Theory and Application to Embodied

1119 Cognitive Systems. Ph.D. Thesis, Indiana University.

1120 Williams, P.L, \& Beer, R.D. 2010. Nonnegative decomposition of multivariate information.

1121 Available from arXiv:1004.2515.

1122 Zhang, R., Zhang, L., Guo, Y., Shi, L., Gai, J., Wang, X., Hu, Y. 2020. Effects of High-

1123 Definition Transcranial Direct-Current Stimulation on Resting-State Functional

1124 Connectivity in Patients with Disorders of Consciousness. Frontiers in Human

1125 Neuroscience, vol. 23: 14:560586. doi: 10.3389/fnhum.2020.560586

1126

1127

1128 